\title{
Continua with partially constrained microstructure
}

\author{
Dedicated to Gianfranco Capriz on the occasion of his 96th birthday
}

Received: 27 January 2021 / Accepted: 21 September 2021 / Published online: 18 October 2021 (C) The Author(s) 2021

\begin{abstract}
The mechanical balance equations for a body with microstructure are derived from an expansion of the general Noll's axiom of frame-indifference that takes into account the behavior of measures of microstructural interactions. Next, we introduce perfect internal constraints and adopt an extended determinism principle to analyze the consequences of their presence. Finally, we define the class of continua with partially constrained microstructure to give a complete dynamical description for a broad family of peculiar materials such as suspensions of rigid rotating granules, pseudo-Cosserat continua and partially constrained micro-spins.
\end{abstract}

Keywords Multiscale models $\cdot$ Invariance $\cdot$ Continua with microstructure $\cdot$ Internal constraints $\cdot$ Balance laws

\section{Introduction}

In continuum mechanics, the possible restrictions to the motion of a material point are represented by the internal constraints, i.e., any device that limits the positions and/or velocities of the points of a material system. Classical examples, extensively studied, are the incompressibility in the dynamics of fluids and the inextensibility in the mechanics of solid elastic materials.

The first complete general mechanical theory of internal constraints was developed by Noll in $\$ 30$ of [68], and was generalized to the thermodynamic variables in $[46,49,53]$. Afterward in [15], the theory was extended to the dynamics of oriented materials, such as Cosserat brothers' continua [22,48], and subsequently, more generally, to the case of continua with microstructure in [17], as well as the diatomic continua are [24,33,40], or the many examples in the essay [6].

At the end, when the microstructural parameters result to be completely constrained to the macrodeformation, we obtain the so-called latent microstructure, which includes some other important types of material, such as continua with voids [43,61], bubbly liquids [8], dilatant granular materials [44,45], Korteweg fluids $[4,28]$.

The present work concerns the use of internal constraints to expand the mechanical theory developed in $[17,49]$ in order to study the essential features of media with a microstructure which is partially constrained. Precisely, we specify, in the list of kinematic order parameters on manifolds which describe the substructures, the local charts that are free, constrained or linked to the macro-motion, respectively.

Communicated by Andreas Öchsner.

P. Giovine $(\bowtie)$

Department of Civil, Energy, Environment and Materials Engineering (DICEAM), Mediterranean University of Reggio Calabria, Reggio Calabria, Italy

E-mail: giovine@unirc.it 
Next, we formulate a principle of extended determinism which permits us to obtain all the pure, i.e., reaction-free, mechanical balance laws for the observed material and, subsequently, the remaining balance equations necessary to determine the corresponding reactions to the constraints.

In the end, we observe that the form of the Cauchy's equation looks like the classical one, but some traditional tenets are abandoned, that is, the stress tensor need not have to be symmetric and can depend on higher time and/or spatial derivatives of displacement.

Afterward, in the last sections of the paper, we use the previous results and consider various general examples of partially constrained theories of microstructured continuous bodies, focusing attention on the subdivision of the mechanical response of materials to deformation into its reactive and determined parts, both in the holonomic and in the anholonomic cases, and we lay down the equations that determine the reactions once the solution of a pure problem of motion has been found.

Furthermore, the peculiarities of the suspensions of rigid rotating granules in a fluid matrix and of pseudoCosserat continua are here explicitly obtained, while we can already recover other different instances for solids with nano-pores and soil mechanics (see, e.g., $[34,41,42]$ ), as well as in the theory of ephemeral continua [12].

\section{Deformations, configuration spaces and motions in materials with microstructure}

We indicate by $\mathcal{B}_{*}$ the regular region (in the sense of fit regions in [59]) of the three-dimensional Euclidean point space $\mathcal{E}^{3}$ occupied by the body in its reference placement. If we denote a generic material element of $\mathcal{B}_{*}$ by $\mathbf{x}_{*}$, a mapping $\tilde{\mathbf{x}}$, acting as

$$
\mathcal{B}_{*} \ni \mathbf{x}_{*} \stackrel{\tilde{\mathbf{x}}}{\longmapsto} \mathbf{x}=\tilde{\mathbf{x}}\left(\mathbf{x}_{*}\right) \in \mathcal{E}^{3}
$$

(and assumed to be one-to-one, continuous and piecewise continuously differentiable), is a deformation: it indicates the current place $\mathbf{x}$ of a generic material element of the body resting at $\mathbf{x}_{*}$ in $\mathcal{B}_{*}$; the current deformed placement of the body is the regular region $\mathcal{B}=\tilde{\mathbf{x}}\left(\mathcal{B}_{*}\right)$.

Moreover, we presume also that $\tilde{\mathbf{x}}$ be orientation preserving and hence this is equivalent to prescribe that, at each $\mathbf{x}_{*}$, the deformation gradient, indicated by $\mathbf{F}$ (namely $\mathbf{F}:=\nabla \tilde{\mathbf{x}}\left(\mathbf{x}_{*}\right)$ ), has positive determinant $\iota$, i.e.,

$$
\iota:=\operatorname{det} \mathbf{F}>0 .
$$

Now, in order to describe the whole mechanical format necessary to analyze the behavior of materials with microstructure, we need to associate then to each material element of the body not only its placement $\mathbf{x}$ in $\mathcal{E}^{3}$ but also a set of $m$ order parameters, its microstate $v$, which is an element of a paracompact differentiable manifold $\mathcal{M}$ of dimension $m$, that we presume here without boundary; hence, we have a map $\tilde{v}$ given by

$$
\mathcal{B}_{*} \ni \mathbf{x}_{*} \stackrel{\tilde{v}}{\longmapsto} v=\tilde{v}\left(\mathbf{x}_{*}\right) \in \mathcal{M},
$$

that we suppose to be continuous and piecewise continuously differentiable.

In this phase, we avoid any particular choice of order parameters that would allow us to take into account peculiar aspects of the morphology of the microstructure of the material, but not others. Furthermore, the geometric properties of a particular manifold $\mathcal{M}$ could have a physical meaning: for example, a connection on $\mathcal{M}$ allows to represent contact micro-interactions acting on the microstructure of a material element of the body and due to microstructural changes in the adjacent elements, instead of some weakly non-local micro-interactions of gradient nature (see $[10,11]$ ), while the assignment of a own micro-inertia to the material microstructure, and so of a peculiar micro-kinetic energy, induces a metric over $\mathcal{M}$; contrariwise, the selection of a particular metric over $\mathcal{M}$ affects the expression of the micro-kinetic energy (see [9,10]). Therefore, now, we do not choose any order parameter nor manifold, which could reduce the generality of the theory.

The space of configurations in a multifield theory is the collection $\mathcal{C}$ of pairs $(\tilde{\mathbf{x}}(\cdot), \tilde{v}(\cdot))$ that may be endowed by the structure of a manifold; its tangent space is indicated by $T \mathcal{C}$, whereas its cotangent space by $T^{*} \mathcal{C}$. Hence, a motion of duration $\bar{\tau}(\bar{\tau}>0)$ for the body is a sufficiently smooth time parametrized curve over $\mathcal{C}$

$$
[0, \bar{\tau}] \ni \tau \longmapsto\left(\tilde{\mathbf{x}}_{\tau}, \tilde{v}_{\tau}\right)=\left(\tilde{\mathbf{x}}\left(\mathbf{x}_{*}, \tau\right), \tilde{v}\left(\mathbf{x}_{*}, \tau\right)\right) \in \mathcal{C}
$$


where we indicate the current placement of a material element, at rest in $\mathbf{x}_{*}$ at time $\tau=0$, and its current microstate by $\tilde{\mathbf{x}}\left(\mathbf{x}_{*}, \tau\right)$ and $\tilde{v}\left(\mathbf{x}_{*}, \tau\right)$, respectively.

Moreover, the velocity fields are given by

$$
\dot{\mathbf{x}}:=\frac{\mathrm{d} \tilde{\mathbf{x}}}{\mathrm{d} \tau}\left(\mathbf{x}_{*}, \tau\right) \in T_{\mathbf{x}} \mathcal{B}, \quad \dot{v}:=\frac{\mathrm{d} \tilde{v}}{\mathrm{~d} \tau}\left(\mathbf{x}_{*}, \tau\right) \in T_{\nu} \mathcal{M},
$$

where $T_{\mathbf{X}} \mathcal{B}$ and $T_{\nu} \mathcal{M}$ are the tangent spaces to $\mathcal{B}$ at $\mathbf{x}$ and to $\mathcal{M}$ at $v$, respectively; of course, the pair $(\dot{\mathbf{x}}(\cdot, \tau), \dot{v}(\cdot, \tau))$ belongs to $T_{(\mathbf{x}, v)} \mathcal{C}$ (the dependance on $\tau$ remaining tacit).

Remark 1 We observe here that each tangent space $T_{v} \mathcal{M}$ is a linear space, while the union $T \mathcal{M}$ of all tangent spaces to $\mathcal{M}$, namely the tangent bundle $T \mathcal{M}=\bigcup_{v \in \mathcal{M}} T_{v} \mathcal{M}$, does not coincide, in general, with a linear space, even if it has the natural structure of a differentiable manifold of dimension $2 m$ whose elements are the pairs $(v, \dot{v})$.

Moreover, the cotangent space $T_{v}^{*} \mathcal{M}$ of $\mathcal{M}$ at $v$, i.e., the dual space of each tangent space $T_{v} \mathcal{M}$, is a linear space of dimension $m$ too, but also the cotangent bundle $T^{*} \mathcal{M}:=\bigcup_{v \in \mathcal{M}} T_{v}^{*} \mathcal{M}$ does not coincide, in general, with a linear space. As well it is endowed with a natural structure of a differentiable manifold of dimension $2 m$ and of a duality pairing; its elements are the pairs $(v, \mathbf{z})$ with the cotangent vector $\mathbf{z} \in T_{v}^{*} \mathcal{M}$ (see, e.g., [52] for a complete description of smooth manifolds).

Let us consider now two observers differing by a rotation of characteristic vector $\mathbf{q}$, with corresponding proper orthogonal tensor

$$
\mathbf{Q}=\exp (-\epsilon \mathbf{q}):=\mathbf{I}-\epsilon \mathbf{q}+\frac{1}{2}(\epsilon \mathbf{q})(\epsilon \mathbf{q})-\ldots,
$$

where exp is the basis of natural logarithms, $\epsilon$ is Ricci's three-dimensional alternating tensor and $\mathbf{I}$ is the identity tensor. These two observers evaluate two different values $v$ and $v_{\mathbf{q}}$ of the order parameters connected by the following relation (see $\S 3$ of [6] or $\S 6$ of [19]):

$$
v_{\mathbf{q}}=v+\mathcal{A q}+o(|\mathbf{q}|)
$$

where $\mathcal{A}$, the Fréchet derivative of $v_{\mathbf{q}}$ at $v$, is the infinitesimal generator of the local action on $\mathcal{M}$ of the group of the proper orthogonal tensors $\mathbf{Q}$ defined by:

$$
\mathcal{A}(v):=\left.\frac{\mathrm{d} v_{\mathbf{q}}}{\mathrm{dq}}\right|_{\mathbf{q}=\mathbf{0}}
$$

$\mathcal{A}$ is a linear operator mapping vectors of $\mathcal{E}^{3}$ into elements of the tangent space of $\mathcal{M}$ at $v$ (i.e., $\mathcal{A} \in$ Hom $\left.\left\{T_{\mathbf{x}} \mathcal{B}, T_{v} \mathcal{M}\right\}\right)$ and, in its matrix representation, has three columns and a number of rows equal to the dimension $m$ of $\mathcal{M}$.

Therefore, in frames that move one with respect to another with translational velocity $\mathbf{c}(\tau)$ and rotational velocity $\dot{\mathbf{q}}(\tau)$ but coincide at the instant of observation, two observers read macro- and micro-velocities related by the following formulae:

$$
\begin{aligned}
\dot{\mathbf{x}}^{\prime} & =\dot{\mathbf{x}}\left(\mathbf{x}_{*}, \tau\right)+\mathbf{c}(\tau)+\dot{\mathbf{q}}(\tau) \times\left(\mathbf{x}\left(\mathbf{x}_{*}, \tau\right)-\mathbf{x}_{0}\right), \\
\dot{v}^{\prime} & =\dot{v}\left(\mathbf{x}_{*}, \tau\right)+\mathcal{A}\left(v\left(\mathbf{x}_{*}, \tau\right)\right) \dot{\mathbf{q}}(\tau),
\end{aligned}
$$

respectively, where $\mathbf{x}_{0}$ is a fixed point chosen arbitrarily in $\mathcal{E}^{3}$ (see equations (2.9) and (2.10) in [20]).

Finally, a complete motion of the body is said rigid if there is an observer for whom both macro- and micro-velocity vanish identically on $\mathcal{B}$, i.e., an observer for which the body appears in complete rest (cf. $\S 6$ of [19]). Hence, the most general rigid velocity distribution for $\mathcal{B}$ (indicated by $\dot{\mathbf{x}}_{R}$ and $\dot{v}_{R}$ ), with translatory speed $\mathbf{c}(\tau)$, constant in space and angular speed $\mathbf{w}(\tau)$, is shown by the expressions:

$$
\dot{\mathbf{x}}_{R}=\mathbf{c}(\tau)+\mathbf{w}(\tau) \times\left(\mathbf{x}_{R}-\mathbf{x}_{0}\right), \quad \dot{v}_{R}=\mathcal{A}\left(v_{R}\right) \mathbf{w}(\tau) .
$$




\section{Balance laws}

In order to deduce the balance equations for materials with microstructure, we prefer to follow Noll's procedure in [58] by requiring the invariance of the outer power with respect to classical changes in spatial observers, rather than to postulate integral balances, and generalize it to interactions of microstructural nature too (see [14]).

If we denote by $\wp\left(\mathcal{B}_{*}\right)$ the set of all subsets $\mathcal{P}$ of $\mathcal{B}_{*}$ with non-null volume measure and the same regularity properties of $\mathcal{B}_{*}$ itself, we call working (or virtual power) a map

$$
\mathcal{W}: \wp\left(\mathcal{B}_{*}\right) \times T \mathcal{C} \longrightarrow \Re
$$

( $\Re$ the set of real numbers) such that: (i) $\mathcal{W}(\cdot, \dot{\mathbf{x}}, \dot{v})$ is additive on disjoint parts and (ii) $\mathcal{W}(\mathcal{P}, \cdot, \cdot)$ is bilinear.

We appeal to known integral representation theorems (see, e.g., [26]), as generalized in [64], for which there is a natural isomorphism between the dual bundle $T_{(\mathbf{x}, v)}^{*} \mathcal{C}$ of $T_{(\mathbf{x}, v)} \mathcal{C}$ and the space of vector-valued measures on $\mathcal{B}_{*}$, i.e., for any couple of virtual velocities $(\dot{\mathbf{x}}, \dot{v})$ for the pair $(\mathbf{x}, v)$, there exist vector-valued measures $m_{\mathcal{B}}$ and $\mu_{\mathcal{B}}$ such that the power of a set of actions on the body $\mathcal{B}_{*}$ takes the form

$$
\mathcal{W}\left(\mathcal{B}_{*}, \dot{\mathbf{x}}, \dot{v}\right)=\int_{\mathcal{B}_{*}}\left(\dot{\mathbf{x}} \cdot d m_{\mathcal{B}}+\left\langle\dot{v}, \mathrm{~d} \mu_{\mathcal{B}}\right\rangle\right),
$$

where $\langle\cdot, \cdot\rangle$ is the natural duality pairing introduced in Remark 1 of Sect. 2, comma 2. Different specifications of those measures imply different models, as standard continua or media with edge interactions (see $[14,60])$. Moreover, if we consider more general tensor-valued measures into the expression (12) of working (depending on the smoothness of velocity fields $(\dot{\mathbf{x}}, \dot{v}))$, then we can introduce "stresses" which may not derive from boundary tractions (as when a physically significant connection cannot be defined on the manifold $\mathcal{M}$ [10]) or that may be non-local [11]: a general introduction to these very subtle questions can be found in $[18,63]$.

For properties (i) and (ii) of working $\mathcal{W}$, the result in (12) can be formally applied to any part $\mathcal{P}$ of the body $\mathcal{B}_{*}$ simply by substituting $\mathcal{P}$ instead of $\mathcal{B}_{*}$ in it and noting that the measures will depend also on $\mathcal{P}$. Afterward, we can deduce balance equations from this expression by invoking an axiom of frame-indifference, if we specify that measures are intended to represent the total external actions on the part $\mathcal{P}$ of the body $\mathcal{B}_{*}$.

We assume that micro-interactions are of bulk and contact nature (as they are in classical continuum mechanics), the latter exerted through the boundary $\partial \mathcal{P}$, and are defined by quantities associated to microvelocities $\dot{v}$, which belong to the tangent space $T_{v} \mathcal{M}$ to $\mathcal{M}$ at $\nu$. Hence, they are elements of the cotangent space $T_{v}^{*} \mathcal{M}$, so that the scalar product by $\dot{v}$ is well defined.

Consequently, to express the power $\mathcal{W}_{\mathcal{P}}^{\text {out }}(\dot{\mathbf{x}}, \dot{v})$ of all external actions we define the measures of interactions acting on the body $\mathcal{B}_{*}$ in the Lagrangian description as follows: at each $\mathbf{x}_{*}$, external bulk macro- and microforces are measured by the covector density per unit volume $\overline{\mathbf{f}} \in T_{\mathbf{x}}^{*} \mathcal{B}\left(\cong \mathfrak{R}^{3}\right)$ and $\bar{\gamma} \in T_{v}^{*} \mathcal{M}$, respectively, including inertial and non-inertial contributions, when relevant; instead, at the boundary $\partial \mathcal{P}$, surface terms are measured by means of the standard first Piola-Kirchhoff stress tensor $\mathbf{P}$ and of the micro-stress tensor $\mathbb{S} \in \operatorname{Hom}\left\{\mathcal{E}^{3}, T_{v}^{*} \mathcal{M}\right\}$, a linear operator with domain $\mathcal{E}^{3}$ and codomain $T_{v}^{*} \mathcal{M}$ such that, on a surface element where $\mathbf{n}$ is the unit vector of the exterior normal to the boundary $\partial \mathcal{P}$ of $\mathcal{P}$, the co-vector $\sigma$ of $T_{v}^{*} \mathcal{M}$,

$$
\sigma:=\mathbb{S} \mathbf{n}
$$

represents the surface actions exerted on the microstructure through the element. A general proof of the existence of the micro-stress tensor on the basis of geometric measure theory might be obtained by using, for different contexts, the results in $[23,57,65]$, but here we only claim the existence of such a tensor.

Therefore, for each arbitrary part $\mathcal{P}$ of $\mathcal{B}_{*}$, the total outer working over $\mathcal{P}$ along $(\mathbf{x}, v)$, with the pair $(\dot{\mathbf{x}}, \dot{v})$ belonging to the relevant tangent space $T_{(\mathbf{x} v)} \mathcal{C}$, is given by the following expression:

$$
\mathcal{W}_{\mathcal{P}}^{\text {out }}(\dot{\mathbf{x}}, \dot{v})=\int_{\mathcal{P}}(\dot{\mathbf{x}} \cdot \overline{\mathbf{f}}+\dot{v} \cdot \bar{\gamma}) \mathrm{d}^{3} \mathbf{x}_{*}+\int_{\partial \mathcal{P}}(\dot{\mathbf{x}} \cdot \mathbf{P n}+\dot{v} \cdot \subseteq \mathbf{n}) \mathrm{d} \mathcal{H}^{2},
$$

where $d^{3} \mathbf{x}_{*}$ is the usual volume measure and $d \mathcal{H}^{2}$ is the two-dimensional Hausdorff measure over $\partial \mathcal{P}$.

Now we are able to deduce balance equations from the following basic axiom of frame-indifference, clearly stated in [58], that we invoke also in presence of measures of microstructural interactions (see, also, $[14,18,54,57])$ : 
The Extended Noll's Axiom. For a dynamical process, the outer working is objective. That is, for any subbody $\mathcal{P}$ of $\mathcal{B}_{*}$ the global working $\mathcal{W}_{\mathcal{P}}^{\text {out }}$ of all actions exerted on $\mathcal{P}$, external to $\mathcal{P}$, is invariant for arbitrary changes in observers (9).

Introducing relations (9) in (14) and using the extended Noll's axiom, we get

$$
\begin{aligned}
0= & \mathcal{W}_{\mathcal{P}}^{\text {out }}\left(\dot{\mathbf{x}}^{\prime}, \dot{v}^{\prime}\right)-\mathcal{W}_{\mathcal{P}}^{\text {out }}(\dot{\mathbf{x}}, \dot{v})=\int_{\mathcal{P}}\left\{\left[\mathbf{c}+\dot{\mathbf{q}} \times\left(\mathbf{x}-\mathbf{x}_{0}\right)\right] \cdot \overline{\mathbf{f}}\right. \\
& +(\mathcal{A} \dot{\mathbf{q}}) \cdot \bar{\gamma}\} \mathrm{d}^{3} \mathbf{x}_{*}+\int_{\partial \mathcal{P}}\left\{\left[\mathbf{c}+\dot{\mathbf{q}} \times\left(\mathbf{x}-\mathbf{x}_{0}\right)\right] \cdot \mathbf{P n}+(\mathcal{A} \dot{\mathbf{q}}) \cdot(\mathbb{S} \mathbf{n})\right\} \mathrm{d} \mathcal{H}^{2} \\
= & \mathbf{c} \cdot\left(\int_{\mathcal{P}} \overline{\mathbf{f}} \mathrm{d}^{3} \mathbf{x}_{*}+\int_{\partial \mathcal{P}} \mathbf{P n} \mathrm{d} \mathcal{H}^{2}\right)+\dot{\mathbf{q}} \cdot\left\{\int_{\mathcal{P}}\left[\left(\mathbf{x}-\mathbf{x}_{0}\right) \times \overline{\mathbf{f}}+\mathcal{A}^{*} \bar{\gamma}\right] \mathrm{d}^{3} \mathbf{x}_{*}\right. \\
& \left.+\int_{\partial \mathcal{P}}\left[\left(\mathbf{x}-\mathbf{x}_{0}\right) \times(\mathbf{P n})+\mathcal{A}^{*}(\mathbb{S} \mathbf{n})\right] \mathrm{d} \mathcal{H}^{2}\right\},
\end{aligned}
$$

for any choice of vectors $\mathbf{c}$ and $\dot{\mathbf{q}}$, where $\mathcal{A}^{*}$ is the adjoint of $\mathcal{A}$ and maps linearly covectors over $\mathcal{M}$ in three-dimensional vectors: in particular, for any vector $\mathbf{v} \in T_{\mathbf{x}} \mathcal{B}$ and covector $\eta \in T_{v}^{*} \mathcal{M}$, we must have that

$$
\left(\mathcal{A}^{*} \eta\right) \cdot \mathbf{v}=\eta \cdot(\mathcal{A v}) .
$$

The arbitrariness of $\mathbf{c}$ and $\dot{\mathbf{q}}$ permits us to write the following integral balance equations for momentum and moment of momentum, namely:

$$
\begin{aligned}
& \int_{\mathcal{P}} \overline{\mathbf{f}} \mathrm{d}^{3} \mathbf{x}_{*}+\int_{\partial \mathcal{P}} \mathbf{P n} \mathrm{d} \mathcal{H}^{2}=\mathbf{0}, \\
& \int_{\mathcal{P}}\left[\left(\mathbf{x}-\mathbf{x}_{0}\right) \times \overline{\mathbf{f}}+\mathcal{A}^{*} \bar{\gamma}\right] \mathrm{d}^{3} \mathbf{x}_{*} \\
& \quad+\int_{\partial \mathcal{P}}\left[\left(\mathbf{x}-\mathbf{x}_{0}\right) \times(\mathbf{P n})+\mathcal{A}^{*}(\mathbb{S} \mathbf{n})\right] \mathrm{d} \mathcal{H}^{2}=\mathbf{0},
\end{aligned}
$$

for any choice of part $\mathcal{P}$ of $\mathcal{B}_{*}$, where, as mentioned already above, $\overline{\mathbf{f}}$ and $\bar{\gamma}$ are presumed to comprise inertia effects, while $\mathbf{0}$ represents the null vector (and covector). The first equation is equivalent to the classical momentum balance equation, while the other one is a generalization of the standard moment of momentum balance, that can be easily recognized when microstructural interactions are absent.

Moreover, the arbitrariness of $\mathcal{P}$ among subbodies of $\mathcal{B}_{*}$, the divergence theorem and the usual techniques of localization imply from (17) the Cauchy's first law of motion

$$
\overline{\mathbf{f}}+\operatorname{Div} \mathbf{P}=\mathbf{0} .
$$

Therefore, taking into account the validity of (19), we obtain from (18) the following relation

$$
\int_{\mathcal{P}}\left[\epsilon\left(\mathbf{F P}^{T}\right)+\mathcal{A}^{*} \bar{\gamma}\right] \mathrm{d}^{3} \mathbf{x}_{*}+\int_{\partial \mathcal{P}} \mathcal{A}^{*}(\mathbb{S} \mathbf{n}) \mathrm{d} \mathcal{H}^{2}=\mathbf{0},
$$

for any fit region $\mathcal{P} \subset \mathcal{B}_{*}$, and hence, as for Eq. (19), we have the local expression of the balance of moment of momentum in presence of micro-interactions:

$$
\epsilon\left(\mathbf{F P}^{T}\right)+\mathcal{A}^{*} \bar{\gamma}+\operatorname{Div}\left(\mathcal{A}^{*} \mathbb{S}\right)=\mathbf{0} .
$$

We observe that the divergence operator Div (.) in Eq. (21) is classically defined only if the manifold $\mathcal{M}$ of the values of the microstructure does not admit an intrinsic (i.e., a "physically significant") connection, as in the case of a liquid with finely distributed gas bubbles or a micro-cracked solid (see §II.C of [54]); when, instead, the manifold $\mathcal{M}$ admits such an intrinsic connection, then the divergence operator must be changed in the covariant divergence $\operatorname{Div} \mathcal{M}(\cdot)$, the result being the same (see equations (3.1-2) of [10] or, also, any general essay on differential geometry): the change is possible without ambiguity only by the use of the intrinsic connection available for $\mathcal{M}$. For example, within the classical model for nematic liquid crystals and for microrigid Cosserat continua, the respective manifolds $\mathcal{M}$ coincide with the projective plane $\mathbf{P}^{2}$ and with the proper orthogonal group $S O(3)$, while the matching intrinsic connections are, respectively, the Levi-Civita 
one derived from the simplest metric, mentioned casually by some Authors (see, e.g., [25]), and the one obtained by the introduction of Euler angles (see $\$ 3$ of [10] and $\$ 11$ of [7]).

Alternatively, we should limit ourselves to the case where $\mathcal{M}$ is a smooth, complete and connected Riemannian manifold, endowed with its geodesic distance [30]. In the following, we will imply the eventual divergence index $\mathcal{M}$ and will use the same notation Div (.) in the two cases.

The vectorial equations (19) and (21) appear insufficient to determine a satisfactory set of evolution equations for the evolving pair $(\mathbf{x}, v)(3+\mathbf{m}$ quantities in all), even if suitable initial and boundary conditions are added to. Nevertheless, by writing the second one in the following form

$$
\epsilon\left(\mathbf{P F}^{T}\right)-\left(\nabla \mathcal{A}^{*}\right) \mathbb{S}=\mathcal{A}^{*}(\bar{\gamma}+\operatorname{Div} \mathbb{S})
$$

we notice that two conditions have to be satisfied to assure its validness $[13,55]$ : the first requires that the difference $\left[\epsilon\left(\mathbf{P F}^{T}\right)-\left(\nabla \mathcal{A}^{*}\right) \mathbb{S}\right]$ be in the range of $\mathcal{A}^{*}$; thus, there exists a covector $\bar{\zeta} \in T_{v}^{*} \mathcal{M}$ such that

$$
\epsilon\left(\mathbf{P F}^{T}\right)=\mathcal{A}^{*} \bar{\zeta}+\left(\nabla \mathcal{A}^{*}\right) \mathbb{S}
$$

which is the known form of the balance equation of "couples" in the theories of continua with microstructure (see, e.g., equation (9.8) of [6]). The second requirement is that

$$
\bar{\gamma}-\bar{\zeta}+\operatorname{Div} \mathbb{S} \in \mathcal{N}
$$

where $\mathcal{N}$ is the null space of $\mathcal{A}^{*}$, i.e., for any covector $\zeta^{\prime} \in \mathcal{N}$, we have that

$$
\bar{\gamma}-\bar{\zeta}+\operatorname{Div} \mathbb{S}=\zeta^{\prime},
$$

and hence the following micro-momentum balance (the so-called Capriz's balance of micro-forces) holds:

$$
\bar{\gamma}-\zeta+\operatorname{Div} \mathbb{S}=\mathbf{0},
$$

where $\zeta:=\bar{\zeta}+\zeta^{\prime}$ represents the internal micro-actions. Therefore:

(a) if the dimension $n$ of $\mathcal{N}$ vanishes, namely if $\mathcal{N} \equiv\{0\}$, then $\zeta^{\prime}=\mathbf{0}$ and $\bar{\zeta}$ is completely determined by Eq. (25): this is a rare event because all microcelerities should be of rigid type (as for microrigid Cosserat continua);

(b) if it happens that $n=m$, hence $\bar{\zeta}$ is not restricted in any manner (as for the theory of continua with finely distributed voids);

(c) if, more generally, $m>n>0$, and so the range of $\mathcal{A}$ does not cover $T_{\nu} \mathcal{M}$ at any $v$, then the last balance (25) seems to introduce a sort of partial indeterminacy in the evaluation of $\bar{\zeta}$, remaining not exhaustive; therefore, we can discard it in favor of an intrinsic one involving only the $(m-n)$ local coordinates in a chart for the manifold $\mathcal{M}$ (see, again, the nematic liquid crystal model, equations (3.3) of [10] and (44) of [54]).

Alternatively, we need to require deeper invariance properties such as a further extension of Noll's axiom to be invariant also under the $n$-dimensional gauge group which comes out from a one-to-one change of observer on the reading of the values $v$ of the microstructure on $\mathcal{M}$ (see $[13,18,63]$ ) or else, when an intrinsic distant parallelism is not available on $\mathcal{M}$ and there is no room to construct the effect of a change of observer because no effect ensues on $v$, a postulate of invariance of the inner working under change of connection on $\mathcal{M}$ (see $\S 5$ of [25]), or again a property of covariance of the balance of substructural interactions, in the sense that it is invariant with respect to the action of the group of diffeomorphisms of $\mathcal{M}$ on itself or on some non-trivial subgroup of it [56].

Finally, the balance (23) still holds if we substitute $\bar{\zeta}$ with the internal micro-actions $\zeta$ because $\mathcal{A}^{*} \bar{\zeta}=$ $\mathcal{A}^{*} \bar{\zeta}+\mathcal{A}^{*} \zeta^{\prime}=\mathcal{A}^{*} \zeta$ and so

$$
\epsilon\left(\mathbf{P F}^{T}\right)=\mathcal{A}^{*} \zeta+\left(\nabla \mathcal{A}^{*}\right) \mathbb{S} .
$$

Therefore, despite the quite different physical circumstances, the derivation from (21) of the balance equations in the form (25) and (26) is always possible, modulo some reduction in the number of variables and/or some invariance request. Moreover, if we apply the tensor of Ricci $\epsilon$ on the left side of Eq. (26), we get

$$
\text { skw }\left(\mathbf{P F}^{T}\right)=\frac{1}{2} \epsilon\left[\mathcal{A}^{*} \zeta+\left(\nabla \mathcal{A}^{*}\right) \mathbb{S}\right] \text {. }
$$


Remark 2 It is important to note from the local balance of moment of momentum in the form (27) that, in general, the tensor $\mathbf{P F}^{T}$ (and so the Cauchy stress tensor $\mathbf{T}:=(\operatorname{det} \mathbf{F})^{-1} \mathbf{P} \mathbf{F}^{T}$ ) is not symmetric in the theory of continua with microstructure. Moreover, an essential implication of (27) is the symmetry of $\mathbf{T}$ not only in the obvious classical theory, when the microstructural actions $\zeta$ and $\mathbb{S}$ are absent, but also if the microstructural parameters are unaffected by gross rotations, i.e., when the operator $\mathcal{A}$ vanishes.

To complete the mechanical picture of the model for the microstructure, we need an explicit expression of the principle of conservation of mass. Suppose that each material element is perfectly identifiable at all times and carries all its mass in its motion, on the contrary we exclude here any phenomenon that implies the creation or vacation of mass (e.g., by radiation or explosions); therefore, we assume that the total mass of the body in the current placement $\mathcal{B}$ is the integral over $\mathcal{B}$ of a mass density $\rho$ and is conserved, as in the classical theory. Thus, if $\rho_{*}$ is the value of $\rho$ in the reference placement $\mathcal{B}_{*}$, the following equality applies

$$
\int_{\mathcal{B}} \rho=\int_{\mathcal{B}_{*}} \rho_{*}
$$

at the same time, similar ones hold for any subbody $\mathcal{P}$, so, along sufficiently regular motions and for smooth density distributions, the local relation follows

$$
\rho\left(\mathbf{x}_{*}, \tau\right) \iota\left(\mathbf{x}_{*}, \tau\right)=\rho_{*}\left(\mathbf{x}_{*}\right)
$$

where $\iota$ is defined in (2), or, equivalently,

$$
\dot{\rho}=-\rho \operatorname{div} \mathbf{v}
$$

where $\operatorname{div}(\cdot)$ is the divergence of $(\cdot)$ in $\mathcal{B}$.

\section{Stress power and objectivity}

We are now able to get the expression for the total inner working $\mathcal{W}_{\mathcal{P}}^{\text {in }}$ for a body with microstructure from the definition of the total external working $\mathcal{W}_{\mathcal{P}}^{\text {out }}$ in (14) making use of the divergence theorem and two balance equations of macro- and micro-momentum (19) and (25), respectively; in fact, it brings us to the following result:

$$
\mathcal{W}_{\mathcal{P}}^{\text {out }}(\dot{\mathbf{x}}, \dot{v})=\int_{\mathcal{P}} w \mathrm{~d}^{3} \mathbf{x}_{*}
$$

where the scalar

$$
w:=\mathbf{P} \cdot \dot{\mathbf{F}}+\zeta \cdot \dot{v}+\mathbb{S} \cdot \nabla \dot{v}
$$

is the net inner working per unit volume in the reference placement $\mathcal{B}_{*}$, the so-called stress power in the Lagrangian description (see, e.g., [17]), while $\mathbf{P}$ and $\mathbb{S}$ must be in $\mathcal{C}^{1}(\mathcal{B}) \cap \mathcal{C}^{0}(\partial \mathcal{B})$.

Therefore, it is noteworthy that, if we define the total inner working as

$$
\mathcal{W}_{\mathcal{P}}^{\text {in }}(\dot{\mathbf{x}}, \dot{v}):=-\int_{\mathcal{P}} w \mathrm{~d}^{3} \mathbf{x}_{*}
$$

we can deduce, from Eq. (31), the following consequence of the Noll's axiom:

Proposition 1 The total working $\mathcal{W}_{\mathcal{P}}$, inner and outer, over each arbitrary part $\mathcal{P}$ of $\mathcal{B}_{*}$ has to vanish for any couple of virtual velocities $(\dot{\boldsymbol{x}}, \dot{\boldsymbol{v}})$ for the pair $(\mathbf{x}, \boldsymbol{v})$ :

$$
\mathcal{W}_{\mathcal{P}}(\dot{\boldsymbol{x}}, \dot{v})\left(:=\mathcal{W}_{\mathcal{P}}^{\text {out }}+\mathcal{W}_{\mathcal{P}}^{\text {in }}\right) \equiv 0, \quad \forall \mathcal{P} \subset \mathcal{B}_{*}, \quad \forall(\dot{\boldsymbol{x}}, \dot{v}) \in T_{(\mathbf{x}, v)} \mathcal{C}
$$


In the following, we shall give a suitable definition of a continuum with microstructure subject to perfect internal kinematical constraints, for which the expression of the stress power $w$ has an essential role. Therefore, we furnish here a version of $w$ which shows clearly its independence from the observer by means of the use of the corotational time derivative $\stackrel{\vee}{ }$, marked here by a superimposed little circle, due to the spin tensor

$$
\mathbf{Y}:=-\operatorname{skw}\left(\dot{\mathbf{F}} \mathbf{F}^{-1}\right),
$$

which is an objective measure of the micro-velocity (see, e.g., \$2.8.2.4 of [50]); in Eq. (35), skw indicates the skew part of the tensor in the round brackets.

Precisely, if we introduce the spin vector $\mathbf{r}$ such that

$$
\mathbf{r}:=-\frac{1}{2} \epsilon\left(\dot{\mathbf{F F}}^{-1}\right)=\frac{1}{2} \epsilon \mathbf{Y}=\frac{1}{2} \operatorname{Curl} \dot{\mathbf{x}},
$$

where the differential operator "Curl" of a vector $\mathbf{u}$ is defined as Curl $\mathbf{u}:=-\epsilon(\nabla \mathbf{u})$, for the skew properties of $\epsilon$ we have the subsequent expression for $\stackrel{\nu}{\nu}$

$$
\stackrel{\circ}{v}=\dot{v}-\mathcal{A r}
$$

(see comments in Remark 2 of $\S 6$ in [6]).

Furthermore, the standard decomposition of the local velocity gradient $\mathbf{L}\left(:=\operatorname{grad} \mathbf{v}=\frac{\partial \mathbf{v}}{\partial \mathbf{x}}\right)$ here takes the following form:

$$
\mathbf{L}=\dot{\mathbf{F}} \mathbf{F}^{-1}=\mathbf{D}-\mathbf{Y}=\mathbf{D}-\epsilon \mathbf{r},
$$

where $\mathbf{D}:=\operatorname{sym}\left(\dot{\mathbf{F}} \mathbf{F}^{-1}\right)$ is the stretching tensor (i.e., the symmetric part of $\mathbf{L}$ ).

At the end, we can obtain the requested objective version of the stress power $w$ in (32) by the use of the balance of moment of momentum (26):

$$
\begin{aligned}
w & =\mathbf{P F}^{T} \cdot \dot{\mathbf{F F}}^{-1}+\zeta \cdot \dot{v}+\mathbb{S} \cdot \nabla \dot{v} \\
& =\operatorname{sym}\left(\mathbf{P F}^{T}\right) \cdot \mathbf{D}-\operatorname{skw}\left(\mathbf{P F}^{T}\right) \cdot(\epsilon \mathbf{r})+\zeta \cdot \dot{v}+\mathbb{S} \cdot \nabla \dot{v} \\
& =\operatorname{sym}\left(\mathbf{P F}^{T}\right) \cdot \mathbf{D}-\left[\mathcal{A}^{*} \zeta+\left(\nabla \mathcal{A}^{*}\right) \mathbb{S}\right] \cdot \mathbf{r}+\zeta \cdot \dot{v}+\mathbb{S} \cdot \nabla \dot{v} \\
& =\operatorname{sym}\left(\mathbf{P F}^{T}\right) \cdot \mathbf{D}+\zeta \cdot(\dot{v}-\mathcal{A} \mathbf{r})+\mathbb{S} \cdot\left[\nabla \dot{v}-(\nabla \mathcal{A})^{t} \mathbf{r}\right] \\
& =\operatorname{sym}\left(\mathbf{P F}^{T}\right) \cdot \mathbf{D}+\zeta \cdot \stackrel{\dot{v}}{ }+\mathbb{S} \cdot \nabla \dot{v}+\left(\mathcal{A}^{*} \mathbb{S}\right) \cdot \nabla \mathbf{r},
\end{aligned}
$$

where, in the last row, $\mathbf{D}, \stackrel{\circ}{\nu}, \nabla \stackrel{\circ}{v}$ and $\nabla \mathbf{r}$ are all frame indifferent; the minor right transposition of exponent ${ }^{t}$ on an $n$-order tensor $\mathbb{A}$ has the following meaning: $\left.\left.\left(\left(\mathbb{A}^{t} \mathbf{a}\right) \mathbf{b}\right) \ldots\right) \mathbf{c}=((\mathbb{A} \mathbf{b}) \mathbf{a}) \ldots\right) \mathbf{c}$, for each triple of vectors $\mathbf{a}, \mathbf{b}$ and $\mathbf{c}$, while the minor left one means that $\left.\left.\left(\left({ }^{t} \mathbb{A} \mathbf{a}\right) \ldots\right) \mathbf{b}\right) \mathbf{c}=((\mathbb{A} \mathbf{a}) \ldots) \mathbf{c}\right) \mathbf{b}$.

\section{Internal constraints in microstructured materials}

In the preceding sections, we tacitly supposed that the pair of variables $(\mathbf{x}, v) \in \mathcal{C}$, as well as their velocity distributions $(\dot{\mathbf{x}}, \dot{v}) \in T_{(\mathbf{x}, v)} \mathcal{C}$, could take arbitrary values for each element of the body $\mathcal{B}$; however, there exists a wide class of microstructured media for which, when an element of $\mathcal{B}$ has reached a certain state, the complete placement $\mathbf{x}$, the microstate $v$, the velocity $\dot{\mathbf{x}}$ or the micro-speed $\dot{v}$ are somehow restricted: as examples, we may think of uniaxial liquid crystals, usually modeled as perfect incompressible fluids with a unit vectorial microstructure, or of Cosserat continua, where their tensorial microstructure is constrained to be a proper orthogonal tensor.

We are allowed to define here the class of continua with perfect internal constraints and adopt a principle of extended determinism in order to analyze the consequences of their presence and to give a full dynamical description for a broad family of such peculiar materials with microstructure. The mechanical theory of internal constraints in media with microstructure, such as those we conceive here, is a non-trivial case of the abstract thermodynamical theory of constrained materials developed in [49], as generalized in [17].

The body $\mathcal{B}$ is said to be internally constrained if, assigned any couple of variables $(\mathbf{x}, v)$ that represent the isothermal state of any material element placed at $\mathbf{x}_{*}$ together with $\mathbf{F}$ and $\nabla v$, the allowed velocity and microvelocity distributions $(\dot{\mathbf{x}}, \dot{v})$ are such that not all values of the objective factors $\mathbf{D}$, in the space of symmetric 
tensors, $\stackrel{\circ}{\nu}$, in the tangent space $T_{v} \mathcal{M}, \nabla \stackrel{\circ}{\nu}$, in the space of linear operators from $T_{x} \mathcal{B}$ into $T_{v} \mathcal{M}$, and $\nabla \mathbf{r}$, between the second-order tensors, are accessible (see, also, §IV.7 of [67] and §9 of [19]).

Thus, we gave reasons to establish the following

Principle of Extended Determinism. For materials with microstructure subject to constraints, each quantity, which in the absence of the constraints themselves is ruled by a constitutive prescription (i.e., $\mathbf{P}, \zeta$ and $\mathbb{S}$ ), is now the direct sum of two components, one active and the other one reactive:

$$
\mathbf{P}=\mathbf{P}_{a}+\mathbf{P}_{r}, \zeta=\zeta_{a}+\zeta_{r}, \mathbb{S}=\mathbb{S}_{a}+\mathbb{S}_{r},
$$

where only the active components $\mathbf{P}_{a}, \zeta_{a}$ and $\mathbb{S}_{a}$ must be specified, through suitable constitutive relations, by the independent kinetic variables. As for the reactive terms $\mathbf{P}_{r}, \zeta_{r}$ and $\mathbf{S}_{r}$, in general, they remain undetermined unless some information on the physical mechanism that causes the constraint is given: in any case, they will depend on the specific process that occurs in the present site $\mathcal{B}$ and their values reduce to fields in the orthogonal complement of the respective active functionals (see, also, equations (5.2) of [15] and (3A.2.5) of [17] in the mechanical theory).

However, as we anticipated before, we will consider the class of bodies with perfect constraints (i.e., internally frictionless) for which, in this purely mechanical context, reactive stress and micro-stresses do no work: namely, the contribution of reactions to the stress power (39) 5 is identically zero for every process allowed by the constraints:

$$
\operatorname{sym}\left(\mathbf{P}_{r} \mathbf{F}^{T}\right) \cdot \mathbf{D}+\zeta_{r} \cdot \stackrel{\circ}{v}+\mathbb{S}_{r} \cdot \nabla \stackrel{v}{ }+\left(\mathcal{A}^{*} \mathbb{S}_{r}\right) \cdot \nabla \mathbf{r}=0,
$$

so that the contribution of reactions $\mathbf{P}_{r}, \zeta_{r}$ and $\mathbb{S}_{r}$ to $w$ is powerless.

In many cases, it is always possible to eliminate the reactive components from the balance equations by using some of Eqs. (19), (25), (27) and (41) and then arrive at a subset of pure equations, which alone are sufficient to study the evolution of $\mathcal{B}$.

Many consequences of assumptions (40) and (41) have been studied in Chap. II of the treatise [6], obtaining a number of special theories, such as bubbly liquids, uni- and bi-axial nematic liquid crystals and Cosserat continua; furthermore, when the microstructural parameters are completely constrained to the macro-motion, only an indirect trace remains of the existence of the microstructure, now said latent: as consequences of the same assumptions, the classical models of the elastic materials of Dunn and Serrin [28], the perfect fluids of Korteweg [4], the materials of Korteweg type [27] or the granular materials with inelastic grains [32] have been obtained.

However, in all the examples some peculiar facts emerge: the Cauchy stress $\mathbf{T}$ can depend on the acceleration gradients, it does not necessarily have to be objective nor, even in these constrained cases, symmetrical.

\section{Continua with partially constrained microstructure}

Here, otherwise, we present a mixed instance in which we suppose to constrain only some order parameters that describe the microstructure, possibly to macro-deformation, while the rest remain free to vary along the physical process we are considering. Therefore, we rearrange their indexes on a local chart of the manifold $\mathcal{M}$ so that the first $p$ parameters, with $0 \leq p \leq m$, are free and the last $(m-p)$ constrained; hence, we have

$$
v=\left(\frac{v^{f}}{v^{c}}\right) \quad \text { with } \quad v^{f}:=\left(\begin{array}{c}
v_{1} \\
\vdots \\
v_{p}
\end{array}\right) \quad \text { and } \quad v^{c}:=\left(\begin{array}{c}
v_{p+1} \\
\vdots \\
v_{m}
\end{array}\right) ;
$$

further, we can think of a finer decomposition of the coordinates supposing that, among the $(m-p)$ constrained parameters, the first $(q-p)$ are connected to the macro-motion, while last $(m-q)$ are not, with $p \leq q \leq m$; thus, in addition, we are able to write

$$
v^{c}=\left(\frac{v^{d}}{v^{e}}\right), \quad \text { with } \quad v^{d}:=\left(\begin{array}{c}
v_{p+1} \\
\vdots \\
v_{q}
\end{array}\right) \quad \text { and } \quad v^{e}:=\left(\begin{array}{c}
v_{q+1} \\
\vdots \\
v_{m}
\end{array}\right) .
$$

For example, the latent microstructure occurs when $p=0$ and $q=m$; instead, if $p=q=0$ the microstructure is constrained in full; finally, when $p=q=m$, the unconstrained case takes place. 
Similarly to parameters $v$, we separate the corresponding components of the fields $\zeta, \mathbb{S}$ and $\mathcal{A}$, e.g., we have from (42)

$$
\zeta=\left(\frac{\zeta^{f}}{\zeta^{c}}\right):=\left(\begin{array}{c}
\zeta_{1} \\
\vdots \\
\zeta_{p} \\
\zeta_{p+1} \\
\vdots \\
\zeta_{m}
\end{array}\right), \quad \mathbb{S}=\left(\frac{\mathbb{S}^{f}}{\mathbb{S}^{c}}\right):=\left(\begin{array}{ccc}
\mathbb{S}_{11} & \mathbb{S}_{12} & \mathbb{S}_{13} \\
\vdots & \vdots & \vdots \\
\mathbb{S}_{p 1} & \mathbb{S}_{p 2} & \mathbb{S}_{p 3} \\
\hline \mathbb{S}_{(p+1) 1} & \mathbb{S}_{(p+1) 2} & \mathbb{S}_{(p+1) 3} \\
\vdots & \vdots & \vdots \\
\mathbb{S}_{m 1} & \mathbb{S}_{m 2} & \mathbb{S}_{m 3}
\end{array}\right)
$$

and, likewise, for $\mathcal{A}$, while, similarly, we could obtain analogous subdecompositions for $(\cdot)^{c}$ fields by the use of definitions (43).

To write the expression of the stress power $w$, we introduce this decomposition into the relations $(39)_{4,5}$ to deduce the following

$$
\begin{aligned}
w= & \operatorname{sym}\left(\mathbf{P F}^{T}\right) \cdot \mathbf{D}+\zeta^{f} \cdot \stackrel{\circ}{v}^{f}+\mathbb{S}^{f} \cdot\left[\nabla \dot{v}^{f}-\left(\nabla \mathcal{A}^{f}\right)^{t} \mathbf{r}\right] \\
& +\zeta^{c} \cdot \stackrel{\circ}{v}^{c}+\mathbb{S}^{c} \cdot \nabla \stackrel{\circ}{v}^{c}+\left[\left(\mathcal{A}^{c}\right)^{*} \mathbb{S}^{c}\right] \cdot \nabla \mathbf{r} .
\end{aligned}
$$

Now, we specify better the general hypothesis for the order parameters $v^{d}$ constrained to the macro-motion:

(•) a set of frictionless holonomic or anholonomic constraints expresses either parameters $v^{d}$ in terms of displacement gradient $F$ and, perhaps, its gradients, or the micro-velocities $\dot{v}^{d}$ in terms of velocity gradient $\nabla v$ and, perhaps, its higher gradients.

Therefore, by applying the principle of extended determinism to these perfect constraints, we obtain, from condition (41),

$$
\begin{aligned}
& \operatorname{sym}\left(\mathbf{P}_{r} \mathbf{F}^{T}\right) \cdot \mathbf{D}+\zeta_{r}^{d} \cdot \stackrel{\circ}{v}^{d}+\mathbb{S}_{r}^{d} \cdot \nabla \stackrel{\circ}{\nu}^{d}+\left[\left(\mathcal{A}^{d}\right)^{*} \mathbb{S}_{r}^{d}\right] \cdot \nabla \mathbf{r}+\zeta_{r}^{f} \cdot \stackrel{\circ}{v}^{f} \\
& +\mathbb{S}_{r}^{f} \cdot\left[\nabla \dot{v}^{f}-\left(\nabla \mathcal{A}^{f}\right)^{t} \mathbf{r}\right]+\zeta_{r}^{e} \cdot \stackrel{\nu}{\nu}^{e}+\mathbb{S}_{r}^{e} \cdot \nabla \stackrel{\nu}{\nu}^{e}+\left[\left(\mathcal{A}^{e}\right)^{*} \mathbb{S}_{r}^{e}\right] \cdot \nabla \mathbf{r}=0,
\end{aligned}
$$

where it can be specified that the first four terms link some micro-reaction to the macro-movement, the next two terms regard free reactions and the last three terms concern other constrained micro-reactions.

Again, a great variety of cases can be dealt with on the basis of the identifications of $v^{f}, v^{d}$ and $v^{e}$ and many special properties can be deduced, rather suggestive of some example of specific cases.

\subsection{Partially constrained elastic materials}

We consider below the general case when the constrained part of the microstate $v^{c}$ is completely determined by the macro-strain $\mathbf{F}$ (i.e., $q=m$ ), a case which includes a certain number of known materials, as we will see in the following:

$$
v^{c}=v^{d}=\omega(\mathbf{F})
$$

this relation represents an internal constraint only if it is objective, and so the following property must be valid

$$
v_{\mathbf{q}}^{d}=\omega(\mathbf{Q F}), \quad \forall \mathbf{Q} \in S O(3),
$$

because the deformation gradient $\mathbf{F}$ is a double vector; Eq. (48) is true for relations (6)-(8) if and only if, in components,

$$
\left(\mathcal{A}^{d}\right)^{\alpha}{ }_{i}=-\epsilon_{i j k} \frac{\partial \omega^{\alpha}}{\partial F_{j J}} F_{k J}
$$

Component notations are specified as follows: the components of the fields in the tangent space $T_{\nu} \mathcal{M}$ of $\mathcal{M}$ at $v$ are marked with Greek exponents, while the Cartesian components of the fields in the Euclidean space are marked with Latin indices, usually in lowercase, except when double vectors are involved (such as $\mathbf{F}$ ) for 
which capital letters are also used; the convention, according to which repeated indices are to be summed, is adopted here and throughout the paper.

Now, if we insert the third-order mixed tensor defined by

$$
\mathcal{O}^{\alpha}{ }_{j k}:=\frac{\partial \omega^{\alpha}}{\partial F_{j J}} F_{k J}
$$

in the condition (49) and use the constraint (47) in the corotational derivative $\stackrel{\circ}{\nu}^{d}$, we have, for relation (38) 3 ,

$$
\stackrel{\circ}{v}^{d}=\left(\dot{v}^{d}-\mathcal{A}^{d} \mathbf{r}\right)=\mathcal{O}(\mathbf{L}+\epsilon \mathbf{r})=\mathcal{O} \mathbf{D} .
$$

Afterward, we substitute relation $(51)_{3}$ in Eq. (46) and use again relation (38) 3 with the fact that

$$
\begin{aligned}
(\nabla \mathbf{L})_{i j H} & =\frac{\partial L_{i j}}{\partial X_{H}}=\frac{\partial}{\partial X_{H}}\left(\frac{\partial v_{i}}{\partial x_{j}}\right)=\frac{\partial}{\partial x_{j}}\left(\frac{\partial v_{i}}{\partial X_{H}}\right) \\
& =\frac{\partial^{2} v_{i}}{\partial X_{H} \partial X_{L}} \frac{\partial X_{L}}{\partial x_{j}}=\left(\nabla^{2} \mathbf{v} \oslash \mathbf{F}^{-1}\right)_{i H j},
\end{aligned}
$$

where the tensor product $\oslash$ between tensors of the third and second orders gives a third-rank tensor field so defined:

$$
(\mathcal{H} \oslash \mathbf{S})_{i G j}=\mathcal{H}_{i G L} \mathrm{~S}_{L j}
$$

therefore, we have

$$
\begin{aligned}
& \operatorname{sym}\left[\mathbf{P}_{r} \mathbf{F}^{T}+\mathcal{O}^{*} \zeta_{r}^{d}+\left(\nabla \mathcal{O}^{*}\right) \mathbb{S}_{r}^{d}\right] \cdot \mathbf{D}+\left[\left(\frac{\mathrm{d} \omega}{\mathrm{d} \mathbf{F}}\right)^{*} \oslash \mathbb{S}_{r}^{d}\right] \cdot \nabla^{2} \mathbf{v} \\
& +\zeta_{r}^{f} \cdot \stackrel{\circ}{v}^{f}+\mathbb{S}_{r}^{f} \cdot\left[\nabla \dot{v}^{f}-\left(\nabla \mathcal{A}^{f}\right)^{t} \mathbf{r}\right]=0
\end{aligned}
$$

where relation (50) has been used and the adjoint operation (of exponent ${ }^{*}$ ) on the third-order mixed tensor $\mathcal{O}$ has the following meaning: $\left(\left(\mathcal{O}^{*} \zeta\right) \mathbf{a}\right) \mathbf{b}=((\mathcal{O} \mathbf{a}) \mathbf{b}) \zeta$, for each triple of vectors $\mathbf{a}, \mathbf{b}$ and covector $\zeta$.

Equation (54) for reactions must be satisfied identically for all velocities $\mathbf{v}$ and micro-velocities $\dot{v}^{f}$ distributions that are not bounded at all from the constraint (47), which also leaves the choice of $\mathbf{D} \in$ Sym, $\nabla^{2} \mathbf{v} \in T_{\mathbf{x}} \mathcal{B} \times \operatorname{Sym}, \mathbf{r} \in T_{\mathbf{x}} \mathcal{B}$ and $\nabla \dot{v}^{f} \in \operatorname{Hom}\left\{T_{\mathbf{x}} \mathcal{B}, T_{\nu} \mathcal{M}\right\}$ totally free, where Sym is the set of symmetric tensors.

The reactions are then characterized by the following requirements:

$$
\begin{aligned}
& \operatorname{sym}\left[\mathbf{P}_{r} \mathbf{F}^{T}+\mathcal{O}^{*} \zeta_{r}^{d}+\left(\nabla \mathcal{O}^{*}\right) \mathbb{S}_{r}^{d}\right]=\mathbf{O}, \\
& \operatorname{rsym}\left[\left(\frac{\mathrm{d} \omega}{\mathrm{d} \mathbf{F}}\right)^{*} \oslash \mathbb{S}_{r}^{d}\right]=\mathbf{O}, \quad \zeta_{r}^{f}=\mathbf{0}, \quad \mathbb{S}_{r}^{f}=\mathbf{O},
\end{aligned}
$$

where $\mathbf{O}$ represents the null tensor of any order, while rsym indicates the symmetric part in the last two indices of a third-rank tensor field, i.e., in components: $\left[\operatorname{rsym}\left(\mathcal{D}^{*} \oslash \mathbb{T}\right)\right]_{i j k}=\left(\mathcal{D}^{*} \oslash \mathbb{T}\right)_{i(j k)}=\frac{1}{2}\left[\mathcal{D}^{\alpha}{ }_{i j} \mathbb{T}_{\alpha k}+\mathcal{D}^{\alpha}{ }_{i k} \mathbb{T}_{\alpha j}\right]$; accordingly, the relative active parts must satisfy the Principle of extended determinism of Sect. 5 , for which we have

$$
\begin{aligned}
& {\left[\mathbf{P}_{a} \mathbf{F}^{T}+\mathcal{O}^{*} \zeta_{a}^{d}+\left(\nabla \mathcal{O}^{*}\right) \mathbb{S}_{a}^{d}\right] \in \mathrm{Sym},} \\
& {\left[\left(\frac{\mathrm{d} \omega}{\mathrm{d} \mathbf{F}}\right)^{*} \oslash \mathbb{S}_{r}^{d}\right]^{T} \mathbf{w} \in \operatorname{Sym}, \forall \text { vector } \mathbf{w},}
\end{aligned}
$$

while, for $(55)_{3,4}, \zeta_{a}^{f}$ and $\mathbb{S}_{a}^{f}$ are free fields in $T_{v}^{*} \mathcal{M}$ and $\operatorname{Hom}\left\{T_{\mathbf{x}} \mathcal{B}, T_{v}^{*} \mathcal{M}\right\}$, respectively.

The final step is to seek the appropriate set of pure, i.e., reaction-free, balance equations, which rule the mechanical evolution of the partially constrained elastic model avoiding the presence of reactive terms. First, 
let us consider the Capriz's balance of micro-forces (25) and split it into the free $(p)$ - and bound $(m-p)$ components, as in (42); moreover, let us apply prescriptions $(40)_{3,4}$ with $(55)_{3,4}$, so that

$$
\bar{\gamma}^{f}-\zeta_{a}^{f}+\operatorname{Div} \mathbb{S}_{a}^{f}=\mathbf{0} \text { and } \zeta_{r}^{d}-\operatorname{Div} \mathbb{S}_{r}^{d}=\bar{\gamma}^{d}-\zeta_{a}^{d}+\operatorname{Div} \mathbb{S}_{a}^{d},
$$

where the former is pure while the latter provides micro-reactions, once complete movement is achieved.

Afterward, in order to analyze the Cauchy's balance of forces (19), we need to study the Piola-Kirchhoff tensor $\mathbf{P}$; in particular, using the decomposition into skew and symmetric parts, Eq. (27) and the relation (40) $)_{1}$, we have

$$
\begin{aligned}
\mathbf{P} F^{T} & =\operatorname{skw}\left(\mathbf{P} F^{T}\right)+\operatorname{sym}\left(\mathbf{P} F^{T}\right) \\
& =\frac{1}{2} \epsilon\left[\mathcal{A}^{*} \zeta+\left(\nabla \mathcal{A}^{*}\right) \mathbb{S}\right]+\operatorname{sym}\left(\mathbf{P}_{a} \mathbf{F}^{T}\right)+\operatorname{sym}\left(\mathbf{P}_{r} \mathbf{F}^{T}\right) .
\end{aligned}
$$

Again, let's decompose the micro-actions, as in (42), apply (55) 3,4 , use Eqs. (49) and (50), in addition to the property of Ricci's tensor, and relation $(55)_{1}$, to obtain

$$
\begin{aligned}
\mathbf{P} F^{T}= & \frac{1}{2} \epsilon\left\{\left(\mathcal{A}^{f}\right)^{*} \zeta_{a}^{f}+\left[\nabla\left(\mathcal{A}^{f}\right)^{*}\right] \mathbb{S}_{a}^{f}\right\}-\operatorname{skw}\left[\mathcal{O}^{*} \zeta^{d}+\left(\nabla \mathcal{O}^{*}\right) \mathbb{S}^{d}\right] \\
& +\operatorname{sym}\left(\mathbf{P}_{a} \mathbf{F}^{*}\right)-\operatorname{sym}\left[\mathcal{O}^{*} \zeta_{r}^{d}+\left(\nabla \mathcal{O}^{*}\right) \mathbb{S}_{r}^{d}\right] \\
= & \frac{1}{2} \epsilon\left\{\left(\mathcal{A}^{f}\right)^{*} \zeta_{a}^{f}+\left[\nabla\left(\mathcal{A}^{f}\right)^{*}\right] \mathbb{S}_{a}^{f}\right\}-\left[\mathcal{O}^{*} \zeta^{d}+\left(\nabla \mathcal{O}^{*}\right) \mathbb{S}^{d}\right] \\
& +\operatorname{sym}\left[\mathbf{P}_{a} \mathbf{F}^{T}+\mathcal{O}^{*} \zeta_{a}^{d}+\left(\nabla \mathcal{O}^{*}\right) \mathbb{S}_{a}^{d}\right] \\
= & \frac{1}{2} \epsilon\left\{\left(\mathcal{A}^{f}\right)^{*} \bar{\gamma}^{f}+\operatorname{Div}\left[\left(\mathcal{A}^{f}\right)^{*} \oslash \mathbb{S}_{a}^{f}\right]\right\}-\mathcal{O}^{*} \bar{\gamma}^{d}-\operatorname{Div}\left(\mathcal{O}^{*} \oslash \mathbb{S}_{r}^{d}\right) \\
& +\operatorname{sym}\left[\mathbf{P}_{a} \mathbf{F}^{T}+\mathcal{O}^{*}\left(\zeta_{a}^{d}-\operatorname{Div} \mathbb{S}_{a}^{d}\right)\right]-\operatorname{skw}\left[\operatorname{Div}\left(\mathcal{O}^{*} \oslash \mathbb{S}_{a}^{d}\right)\right],
\end{aligned}
$$

where also balances (57) are used in the last equality.

Because of relations $(55)_{2}$ and $(56)_{2}$, the divergence of the last two terms of this expression vanishes, therefore the pure equation of Cauchy, in Lagrangian formulation, is

$$
\overline{\mathbf{f}}+\operatorname{Div} \hat{\mathbf{P}}=\mathbf{0},
$$

where the following reaction-free expression for the Piola-Kirchhoff stress tensor $\hat{\mathbf{P}}$ applies

$$
\begin{aligned}
\hat{\mathbf{P}}= & \left\{\operatorname{sym}\left[\mathbf{P}_{a} \mathbf{F}^{T}+\mathcal{O}^{*}\left(\zeta_{a}^{d}-\operatorname{Div} \mathbb{S}_{a}^{d}\right)\right]-\mathcal{O}^{*} \bar{\gamma}^{d}\right. \\
& \left.+\frac{1}{2} \epsilon\left\{\left(\mathcal{A}^{f}\right)^{*} \bar{\gamma}^{f}+\operatorname{Div}\left[\left(\mathcal{A}^{f}\right)^{*} \oslash \mathbb{S}_{a}^{f}\right]\right\}\right\} \mathbf{F}^{-T} \\
= & \mathbf{P}_{a}-\frac{\mathrm{d} \omega^{T}}{\mathrm{~d} \mathbf{F}}\left(\bar{\gamma}^{d}-\zeta_{a}^{d}+\operatorname{Div} \mathbb{S}_{a}^{d}\right) \\
& +\left\{\frac{1}{2} \epsilon\left\{\left(\mathcal{A}^{f}\right)^{*} \bar{\gamma}^{f}+\operatorname{Div}\left[\left(\mathcal{A}^{f}\right)^{*} \oslash \mathbb{S}_{a}^{f}\right]\right\}\right\} \mathbf{F}^{-T}
\end{aligned}
$$

and where, in the last equivalence of this equation, we used relations (56) $)_{1}$ and (50). $\hat{\mathbf{P}}$ will be the object of a constitutive prescription: we observe that the resulting Cauchy's tensor $\mathbf{T}\left(:=(\operatorname{det} \mathbf{F})^{-1} \hat{\mathbf{P}} \mathbf{F}^{T}\right)$ is not necessarily symmetric-valued, in general.

To conclude, only the active components $\mathbf{P}_{a}, \zeta_{a}^{d}, \mathbb{S}_{a}^{d}, \zeta_{a}^{f}$ and $\mathbb{S}_{a}^{f}$ of the unknown constitutive fields highlight in the Cauchy equation (60), with $\hat{\mathbf{P}}$ given by (61), and in the free part of the Capriz micro-momentum equation $(57)_{1}$ : these are all the field equations governing the mechanical processes possible in any partially constrained elastic material.

Once a solution to the macro- and micro-motion has been found, Eqs. (19) and (57) 2 , together with (55), determine the reactions corresponding to the constraints within the intrinsic limits deriving from a partial identity of the effects of the fields $\zeta_{r}^{d}$ and Div $\mathbb{S}_{r}^{d}$ and due to their indeterminacy left by Eq. $(57)_{2}$ itself, as discussed in §3, Remark 1, of [15] (see, also, §205 and §227 of [69]). 
Remark 3 A peculiar case of partially constrained elastic materials is the model for the perfect Korteweg fluid by Dunn and Serrin $[4,28]$, when it is assumed that the free coordinates $v^{f}$ are absent, i.e., when $p=0$; therefore, all the fields with superscript ${ }^{f}$ in Sect. 6.1 disappear: this body also represents a continuum with latent microstructure.

\subsection{Partially constrained micro-spins}

Alternatively, we consider the peculiar case in which $q=p$ and the constrained part of the micro-motion $v^{c}$ is forced to be locally of the spin type, so that only micro-speed distributions $\dot{v}^{c}$ of the rigid type, as in $(10)_{2}$, are allowed with an arbitrary choice of the vector field $\mathbf{w}$, but without a condition of global rigidity:

$$
\dot{v}^{c}=\dot{v}^{e}=\mathcal{A}^{e} \mathbf{w}\left(\mathbf{x}_{*}, \tau\right),
$$

where the choice of the vector field $\mathbf{w}$ is arbitrary; therefore, the condition (46) becomes now

$$
\begin{aligned}
& \operatorname{sym}\left(\mathbf{P}_{r} \mathbf{F}^{T}\right) \cdot \mathbf{D}+\zeta_{r}^{f} \cdot \stackrel{\circ}{v}^{f}+\mathbb{S}_{r}^{f} \cdot\left[\nabla \dot{v}^{f}-\left(\nabla \mathcal{A}^{f}\right)^{t} \mathbf{r}\right] \\
& \quad+\left\{\left(\mathcal{A}^{e}\right)^{*} \zeta_{r}^{e}+\left[\nabla\left(\mathcal{A}^{e}\right)^{*}\right] \mathbb{S}_{r}^{e}\right\} \cdot(\mathbf{w}-r)+\left[\left(\mathcal{A}^{e}\right)^{*} \mathbb{S}_{r}^{e}\right] \cdot \nabla \mathbf{w}=0 .
\end{aligned}
$$

Thus, the macro-motion is not constrained at all, as well as the first $p$-parameters $v^{f}$ of the micro-motion, and hence the symmetric tensor $\mathbf{D}$, the vector $\dot{\nu}^{f}$ in $T_{\nu} \mathcal{M}$ and the homomorphism $\left[\nabla \dot{v}^{f}-\left(\nabla \mathcal{A}^{f}\right)^{t} \mathbf{r}\right]$ remain totally free in (63), whereas the factors in parentheses, in the second line, are free only to the extent that the vector $(\mathbf{w}-r)$ and the second-order tensor $\nabla \mathbf{w}$ are locally free.

The following conditions ensue

$$
\begin{aligned}
& \operatorname{sym}\left(\mathbf{P}_{r} \mathbf{F}^{T}\right)=\mathbf{O}, \quad \zeta_{r}^{f}=\mathbf{0}, \quad \mathbb{S}_{r}^{f}=\mathbf{O}, \\
& \left(\mathcal{A}^{e}\right)^{*} \zeta_{r}^{e}+\left[\nabla\left(\mathcal{A}^{e}\right)^{*}\right] \mathbb{S}_{r}^{e}=\mathbf{O}, \quad\left(\mathcal{A}^{e}\right)^{*} \mathbb{S}_{r}^{e}=\mathbf{O} ;
\end{aligned}
$$

in parallel, as stated by the Principle of extended determinism, the active parts satisfy

$$
\operatorname{skw}\left(\mathbf{P}_{a} \mathbf{F}^{T}\right)=\mathbf{O},
$$

while $\zeta_{a}^{f}, \mathbb{S}_{a}^{f},\left\{\left(\mathcal{A}^{e}\right)^{*} \zeta_{a}^{e}+\left[\nabla\left(\mathcal{A}^{e}\right)^{*}\right] \mathbb{S}_{a}^{e}\right\}$ and $\left[\left(\mathcal{A}^{e}\right)^{*} \mathbb{S}_{a}^{e}\right]$ are free fields.

When we consider the conditions (64) $)_{1-4}$ of the reactions together with the balance (27) and the decompositions (40), we infer that

$$
\begin{aligned}
\mathbf{P F} & \operatorname{sym}\left(\mathbf{P F}^{T}\right)+\operatorname{skw}\left(\mathbf{P F}^{T}\right) \\
= & \operatorname{sym}\left(\mathbf{P}_{a} \mathbf{F}^{T}\right)+\frac{1}{2} \epsilon\left[\mathcal{A}^{*} \zeta+\left(\nabla \mathcal{A}^{*}\right) \mathbb{S}\right] \\
= & \operatorname{sym}\left(\mathbf{P}_{a} \mathbf{F}^{T}\right)+\frac{1}{2} \epsilon\left\{\mathcal{A}^{*} \zeta_{a}+\left(\nabla \mathcal{A}^{*}\right) \mathbb{S}_{a}+\left(\mathcal{A}^{f}\right)^{*} \zeta_{r}^{f}+\left[\nabla\left(\mathcal{A}^{f}\right)^{*}\right] \mathbb{S}_{r}^{f}\right. \\
& \left.+\left(\mathcal{A}^{e}\right)^{*} \zeta_{r}^{e}+\left[\nabla\left(\mathcal{A}^{e}\right)^{*}\right] \mathbb{S}_{r}^{e}\right\} \\
= & \operatorname{sym}\left(\mathbf{P}_{a} \mathbf{F}^{T}\right)+\frac{1}{2} \epsilon\left\{\mathcal{A}^{*} \zeta_{a}+\left(\nabla \mathcal{A}^{*}\right) \mathbb{S}_{a}\right\}
\end{aligned}
$$

where again we express the Piola-Kirchhoff stress tensor $\mathbf{P}$ only in terms of its constituent components, which makes the Cauchy's equation (19) pure.

The evolution of the microstructure depends still on the fields $v^{f}$ and $\mathbf{w}$ and, to obtain the pure equations for them too, we divide the Capriz balance (25), as in the previous section, into the free $(p)$ - and constrained $(m-p)$-components, so that

$$
\bar{\gamma}^{f}-\zeta_{a}^{f}+\operatorname{Div} \mathbb{S}_{a}^{f}=\mathbf{0} \text { and } \zeta_{r}^{e}-\operatorname{Div} \mathbb{S}_{r}^{e}=\bar{\gamma}^{e}-\zeta_{a}^{e}+\operatorname{Div} \mathbb{S}_{a}^{e},
$$

where the former is again pure for $(64)_{2,3}$, while it is necessary to apply the operator $\left(\mathcal{A}^{e}\right)^{*}$ on both sides of the latter equation in order to take advantage of the conditions $(64)_{4,5}$, for which

$$
\left(\mathcal{A}^{e}\right)^{*}\left(\bar{\gamma}^{e}-\zeta_{a}^{e}+\operatorname{Div} \mathbb{S}_{a}^{e}\right)=\mathbf{0}
$$


is the last pure equation for $\mathbf{w}$; we observe that the pure covector $\left(\bar{\gamma}^{e}-\zeta_{a}^{e}+\operatorname{Div} \mathbb{S}_{a}^{e}\right)$ must belong to the null space of $\left(\mathcal{A}^{e}\right)^{*}$. give

Again, once a solution to the motions has been obtained, relations (27) and (64) $)_{1-4}$, together with (65),

$$
\begin{aligned}
\mathbf{P}_{r} & =\left[\operatorname{sym}\left(\mathbf{P}_{r} \mathbf{F}^{T}\right)+\operatorname{skw}\left(\mathbf{P}_{r} \mathbf{F}^{T}\right)\right] \mathbf{F}^{-T} \\
& =\frac{1}{2}\left\{\epsilon\left[\mathcal{A}^{*} \zeta_{a}+\left(\nabla \mathcal{A}^{*}\right) \mathbb{S}_{a}\right]\right\} \mathbf{F}^{-T},
\end{aligned}
$$

while the other reactions to constraints are determined by $(64)_{2,3}$ and $(67)_{2}$, always within the intrinsic limits discussed at the end of the previous subsection regarding the difference $\left(\zeta_{r}^{e}-\operatorname{Div} \mathbb{S}_{r}^{e}\right)$.

Remark 4 As an example of a partially constrained micro-spin, we can think of a bubbly uniaxial liquid crystal where $v^{f} \in(0,1)$ is the bubble fraction, which is not affected by rigid rotations and $\mathcal{A}^{f}=0$, while $v^{e}$ is a unit vector $\mathbf{d}$, for which $\dot{\mathbf{d}}=\mathbf{w} \times \mathbf{d}=(\epsilon \mathbf{d}) \mathbf{w}$ and $\mathcal{A}^{e}$ is the second-order tensor $\epsilon \mathbf{d}$.

In this case, the representation (66) reduces to

$$
\mathbf{P} F^{T}=\operatorname{sym}\left(\mathbf{P}_{a} \mathbf{F}^{T}\right)+\operatorname{skw}\left[\mathbf{d} \otimes \zeta_{a}^{e}+(\nabla \mathbf{d})\left(\mathbb{S}_{a}^{e}\right)^{T}\right],
$$

where also $\zeta_{a}^{e}$ is a vector and $\mathbb{S}_{a}^{e}$ a second-order tensor. In the pure equation for the void fraction of bubbles $(67)_{1}, \bar{\gamma}^{f}$ and $\zeta_{a}^{f}$ are scalars and $\mathbb{S}_{a}^{f}$ is a vector, while the pure equation for the angular velocity (68) is now

$$
\left(\bar{\gamma}^{e}-\zeta_{a}^{e}+\operatorname{Div} \mathbb{S}_{a}^{e}\right) \times \mathbf{d}=\mathbf{0},
$$

i.e., the quantity in round brackets is a vector parallel to the unit vector $\mathbf{d}$ (see, also, [17]).

\subsection{Partially constrained affine microstructure}

Here, each material element of a body with affine microstructure is, in general, capable of an affine strain, distinct from (and independent of) the macroscopic one, and also from neighboring elements (as is the case with granular materials). The order parameter $v$ is a second-rank tensor field $\mathbf{G}$ with positive determinant describing the local affine structure, that can also be considered as a double vector, such as $\mathbf{F}$ : accordingly, we will indicate its Cartesian components with one lower and one upper case index; therefore, a rigid rotation of the whole body, characterized by a proper orthogonal tensor $\mathbf{Q}$ in (7), changes the values of the field $\mathbf{G}$ as $\mathbf{G}_{q}=\mathbf{Q}^{T} \mathbf{G}$ (see, also, [29,31]).

Therefore, the infinitesimal generator $\mathcal{A}$ of the group of rotations on the microstructure $\mathbf{G}$ in $\mathrm{Lin}^{+}$is now a third-order tensor field with components

$$
\mathcal{A}_{i J k}=\epsilon_{i m k} \mathrm{G}_{m J}
$$

(see $\$ 2$ of [41]), like the micro-stress $\mathbb{S}$, while the co-vectors $\zeta$ and $\bar{\gamma}$ are also second-order tensors (denoted, respectively, by the symbols $\mathbf{K}$ and $\mathbf{C}$ ). Therefore, the balance of moment of momentum (27) is now

$$
\operatorname{skw}\left(\mathbf{P F}^{T}\right)=\operatorname{skw}\left(\mathbf{G K}^{T}+\nabla \mathbf{G} \odot \mathbb{S}\right),
$$

where the property of the Ricci's alternating tensor $\epsilon$, for which $\frac{1}{2} \epsilon(\epsilon(\cdot))=$ skw $(\cdot)$, has been used; moreover, the tensor product $\odot$ between third-rank tensors has the following components

$$
\left(\mathbb{G} \odot \mathbb{S}_{i j}:=\mathbb{G}_{i H K} \mathbb{S}_{j H K}\right.
$$

Furthermore, we introduce the local wrenching $\mathbf{W}$ (decomposing it as the velocity gradient $\mathbf{L}$ in Eq. (38)) and the wrenching gradient $\mathrm{W}$ :

$$
\mathbf{W}:=\dot{\mathbf{G}} \mathbf{G}^{-1}=\tilde{\mathbf{D}}-\tilde{\mathbf{Y}}, \quad W:=\nabla \mathbf{W}
$$


where $\tilde{\mathbf{D}}:=\operatorname{sym}\left(\dot{\mathbf{G}} \mathbf{G}^{-1}\right)$ and $\tilde{\mathbf{Y}}:=-$ skw $\left(\dot{\mathbf{G}} \mathbf{G}^{-1}\right)$ are the micro-stretching and micro-spin tensors, respectively, for which the stress power $w$ in the expression (39) 4 reduces to the following

$$
\begin{aligned}
w= & \operatorname{sym}\left(\mathbf{P F}^{T}\right) \cdot \mathbf{D}+\mathbf{K} \cdot(\dot{\mathbf{G}}+\mathbf{Y G})+\mathbb{S} \cdot\left\{\nabla \dot{\mathbf{G}}-\left[(\nabla \mathbf{G})^{T} \oslash \mathbf{Y}\right]^{T}\right\} \\
= & \operatorname{sym}\left(\mathbf{P F}^{T}\right) \cdot \mathbf{D}+\left(\mathbf{K G}^{T}+\mathbb{S} \odot \nabla \mathbf{G}\right) \cdot(\mathbf{W}+\mathbf{Y})+\left(\mathbb{S}^{t} \oslash \mathbf{G}^{T}\right) \cdot \mathbb{W}^{t} \\
= & \operatorname{sym}\left(\mathbf{P F}^{T}\right) \cdot \mathbf{D}+\operatorname{sym}\left(\mathbf{K G}^{T}+\mathbb{S} \odot \nabla \mathbf{G}\right) \cdot \tilde{\mathbf{D}} \\
& +\operatorname{skw}\left(\mathbf{K G}^{T}+\mathbb{S} \odot \nabla \mathbf{G}\right) \cdot(\mathbf{Y}-\tilde{\mathbf{Y}})+\left(\mathbb{S}^{t} \oslash \mathbf{G}^{T}\right) \cdot \mathbb{W}^{t},
\end{aligned}
$$

where the relations $(36)_{2}$ and $(75)_{1,2}$ have been used, respectively, in the first, second and third rows. In the last line $(76)_{3}$, the symmetric tensors $\mathbf{D}$ and $\tilde{\mathbf{D}}$, the skew-symmetric tensor $(\mathbf{Y}-\tilde{\mathbf{Y}})$ and the third-rank wrenching gradient $\mathbb{W}$ are all frame indifferent.

The general constraint that we now consider is an affine microdeformation with constrained stretching, a truly anholonomic case as it does not necessarily imply constrained microdeformations. In particular, we set

$$
\operatorname{sym}(\mathbf{L}-W)=\mathbf{D}-\tilde{\mathbf{D}}=\mathbf{O},
$$

i.e., affine microdeformations are only allowed when the micro- and macro-stretching are equal.

Furthermore, the characterization (41) for reaction fields is now

$$
\begin{aligned}
& \operatorname{sym}\left(\mathbf{P}_{r} \mathbf{F}^{T}+\mathbf{K}_{r} \mathbf{G}^{T}+\mathbb{S}_{r} \odot \nabla \mathbf{G}\right) \cdot \mathbf{D} \\
& \quad+\operatorname{skw}\left(\mathbf{K}_{r} \mathbf{G}^{T}+\mathbb{S}_{r} \odot \nabla \mathbf{G}\right) \cdot(\mathbf{Y}-\tilde{\mathbf{Y}})+\left(\mathbb{S}_{r}^{t} \oslash \mathbf{G}^{T}\right) \cdot \mathbb{W}^{t}=\mathbf{O},
\end{aligned}
$$

for all the choices of $\mathbf{D} \in \operatorname{Sym},(\mathbf{Y}-\tilde{\mathbf{Y}}) \in \operatorname{Skw}, \mathbf{G} \in \operatorname{Lin}$ and $\mathbb{W} \in \operatorname{Lin}_{3}$, where Skw is the set of skewsymmetric tensors, Lin the space of second-order tensors identified with linear transformations of $T_{x} \mathcal{B}$ into itself and $\mathrm{Lin}_{3}$ is likewise the space of third-order tensors (linear transformations of ordinary vector space $T_{x} \mathcal{B}$ into Lin, or of Lin into $T_{x} \mathcal{B}$ ). Therefore, we have

$$
\begin{aligned}
& \operatorname{sym}\left(\mathbf{P}_{r} \mathbf{F}^{T}+\mathbf{K}_{r} \mathbf{G}^{T}+\mathbb{S}_{r} \odot \nabla \mathbf{G}\right)=\mathbf{O}, \\
& \operatorname{skw}\left(\mathbf{K}_{r} \mathbf{G}^{T}+\mathbb{S}_{r} \odot \nabla \mathbf{G}\right)=\mathbf{O}, \quad \mathbb{S}_{r}=\mathbf{O},
\end{aligned}
$$

and, consequently,

$$
\begin{aligned}
& \left(\mathbf{P}_{a} \mathbf{F}^{T}+\mathbf{K}_{a} \mathbf{G}^{T}+\mathbb{S}_{a} \odot \nabla \mathbf{G}\right) \in \mathrm{Sym} \\
& \left(\mathbf{K}_{a} \mathbf{G}^{T}+\mathbb{S}_{a} \odot \nabla \mathbf{G}\right) \in \mathrm{Skw}, \quad \mathbb{S}_{a} \in \operatorname{Lin}_{3}
\end{aligned}
$$

at the end we obtain the following constitutive restrictions

$$
\begin{aligned}
& \left(\mathbf{P}_{a} \mathbf{F}^{T}\right) \in \operatorname{Sym}, \quad\left(\mathbf{K}_{a} \mathbf{G}^{T}+\mathbb{S}_{a} \odot \nabla \mathbf{G}\right) \in \mathrm{Skw}, \quad \mathbb{S}=\mathbb{S}_{a} \in \mathrm{Lin}_{3}, \\
& \operatorname{sym}\left(\mathbf{P}_{r} \mathbf{F}^{T}+\mathbf{K}_{r} \mathbf{G}^{T}\right)=\mathbf{O}, \quad \operatorname{skw}\left(\mathbf{K}_{r} \mathbf{G}^{T}\right)=\mathbf{O} .
\end{aligned}
$$

Proceeding as in the previous sections, we get

$$
\begin{aligned}
\mathbf{P F}^{T} & =\operatorname{sym}\left(\mathbf{P F}^{T}\right)+\operatorname{skw}\left(\mathbf{P} \mathbf{F}^{T}\right) \\
& =\operatorname{sym}\left(\mathbf{P}_{a} \mathbf{F}^{T}\right)+\operatorname{sym}\left(\mathbf{P}_{r} \mathbf{F}^{T}\right)-\operatorname{skw}\left(\mathbf{K} G^{T}+\subseteq \odot \nabla \mathbf{G}\right) \\
& =\operatorname{sym}\left(\mathbf{P}_{a} \mathbf{F}^{T}\right)-\operatorname{sym}\left(\mathbf{K}_{r} \mathbf{G}^{T}\right)-\operatorname{skw}\left(\mathbf{K}_{a} \mathbf{G}^{T}+\mathbb{S}_{a} \odot \nabla \mathbf{G}\right),
\end{aligned}
$$


where we used Eqs. (73), (40) and (81) $3,4,5$; the balance of micro-forces is now

$$
\mathbf{C}-\mathbf{K}+\operatorname{Div} \mathbb{S}=\mathbf{O},
$$

and multiplying the left side from the right by the operator $\mathbf{G}^{T}$ and rearranging the addenda using relations (81), we have

$$
\operatorname{sym}\left(\mathbf{K}_{r} \mathbf{G}^{T}\right)+\operatorname{skw}\left(\mathbf{K}_{a} \mathbf{G}^{T}+\mathbb{S}_{a} \odot \nabla \mathbf{G}\right)=\mathbf{C G}^{T}+\operatorname{Div}\left[\left(\mathbb{S}_{a}^{t} \oslash \mathbf{G}^{T}\right)^{t}\right],
$$

so, substituting in (82), it is

$$
\mathbf{P}=\left\{\operatorname{sym}\left(\mathbf{P}_{a} \mathbf{F}^{T}\right)-\mathbf{C G}^{T}-\operatorname{Div}\left[\left(\mathbb{S}_{a}^{t} \oslash \mathbf{G}^{T}\right)^{t}\right]\right\} \mathbf{F}^{-T}
$$

and the Cauchy's equation (19) for a continuum with affine microdeformation with constrained stretching becomes pure. The other pure equation is obtained by taking the skew part of Eq. (84)

$$
\operatorname{skw}\left[\left(\mathbf{C}-K_{a}+\operatorname{Div} \mathbb{S}_{a}\right) \mathbf{G}^{T}\right]=\mathbf{O} .
$$

The only left reaction $\mathbf{K}_{r}$ is obtained from Eqs. (83) and (81) 3

$$
\mathbf{K}_{r}=\mathbf{C}-\mathbf{K}_{a}+\operatorname{Div} \mathbb{S}_{a} .
$$

Remark 5 We observe that the anholonomic constraint (77) of bound stretching $\mathbf{D}=\tilde{\mathbf{D}}$ does not necessarily give us the possibility to identify kinematical parameters $v^{f}$ and $v^{c}$, as defined in (42), even if the microstructure itself results partially constrained (see, also, [15]).

\section{Specific examples}

\subsection{Suspension of rigid granules in a fluid matrix}

In the study of the mechanics of a large number of discrete inelastic particles at relatively high concentrations and with interstices filled with a fluid of negligible mass (as it is the case of soil without cohesion, such as sand with rough surface grains, or of fluidized particulate beds), we must introduce two distinct features to describe the micro-motion: (a) the volume distribution function of the solid granular constituent $\xi$ (firstly introduced in [45]), namely the volume fraction of the solid grains with values on the real interval $(0,1)$ and correlated, in soil mechanics terminology, to the porosity $n$ by the relation $n=1-\xi$ (see, also, [37,44]); (b) the rotation of the rigid granules relative to each other, identified by a proper orthogonal tensor $\mathbf{R}^{\prime}$ not necessarily related to the macro-rotation $\mathbf{R}$ of the body itself (see, e.g., [1,21,39,51]).

Point (a) is better understood if we introduce the proper mass density $\rho_{m}$ of a typical suspended grain in $\mathcal{B}$, which corresponds to the mass density of the granule itself, and the fact that the fluid mass density is considered negligible compared to $\rho_{m}$, then the bulk mass density $\rho$ of the material element equals $\rho_{m}$ times the volume fraction $\xi$ of the grains

$$
\rho=\rho_{m} \xi
$$

Moreover, the hypothesis of rigid granules implies that $\rho_{m}$ is constant, i.e., $\rho_{m}=\rho_{m_{*}}$ (the value in the reference placement $\mathcal{B}_{*}$ of the material), therefore, the conservation of mass (29) reduces to a constraint for the parameter $\xi$

$$
\xi \iota=\xi_{*} ;
$$

in fact, $\xi$ depends only on the determinant $\iota$ of the deformation gradient $\mathbf{F}$ and we can define the functional $\omega$ in (47) as

$$
\omega=\xi_{*} l^{-1}
$$


and so the total volume change of the granular material with inelastic grains is only due to the increase or decrease in the volume of interstitial voids: this phenomenon represents the dilatancy introduced by Reynolds [62] for which, although the volume of granules remains constant, the total volume of the granular medium the total volume of the granular medium can vary, as can be seen from the fact that, in general, $\operatorname{div} \mathbf{v}(=\operatorname{tr} \mathbf{L})$ is not equal to zero for Eqs. (30) and (88) with $\rho_{m}$ constant:

$$
\operatorname{div} \mathbf{v}=-\rho^{-1} \dot{\rho}=-\xi^{-1} \dot{\xi} \neq 0
$$

(see, also, [2]).

The balance equations of motion can be obtained directly from Sect. 6.1 by identifying the parameters $v^{d}$ with $\xi$ and $v^{f}$ with $\mathbf{R}^{\prime}$, except that for the expression of the first Capriz micro-momentum equation (57) 1 because here the corotational derivative $\stackrel{\circ}{\nu}^{f}$ depends on the skew-symmetric spin tensor $\mathbf{Y}$ and so the reaction condition (54) is modified as follows.

In fact, we have that the free infinitesimal generator $\mathcal{A}^{f}$ is now the following third-order tensor field

$$
\mathcal{A}^{f}=-\left(\epsilon \oslash \mathbf{R}^{\prime}\right)^{t}
$$

for the definition (8) (see, also, §23 of [6]); therefore, for (37) and (36) 2 , it is

$$
\stackrel{\circ}{v}^{f}=\dot{\mathbf{R}}^{\prime}+\left(\epsilon \oslash \mathbf{R}^{\prime}\right)^{t} \mathbf{r}=\dot{\mathbf{R}}^{\prime}-(\epsilon \mathbf{r}) \mathbf{R}^{\prime}=\dot{\mathbf{R}}^{\prime}-\mathbf{Y} \mathbf{R}^{\prime}
$$

and moreover, in components,

$$
\begin{aligned}
{\left[\left(\nabla \mathcal{A}^{f}\right)^{t} \mathbf{r}\right]_{i H L} } & =-\left[\nabla\left(\epsilon \oslash \mathbf{R}^{\prime}\right)^{t}\right] \mathbf{r}_{k}=(\epsilon \mathbf{r})_{i j} \mathbf{R}_{j H, L}^{\prime} \\
& =\mathbf{Y}_{i j} \mathbf{R}_{j H, L}^{\prime}=-\left[\left(\nabla \mathbf{R}^{\prime}\right)^{T} \oslash \mathbf{Y}\right]_{i H L}^{T}
\end{aligned}
$$

finally, we observe that also the micro-stress $\mathbb{S}^{f}$ is a third-rank tensor, while the co-vectors $\zeta^{f}$ and $\bar{\gamma}^{f}$ are second-order tensor fields (denoted, respectively, by the symbols $\mathbf{K}^{f}$ and $\overline{\mathbf{C}}^{f}$ ).

In addition, we precise that

$$
\frac{\mathrm{d} \omega}{\mathrm{d} \mathbf{F}}=-\xi \mathbf{F}^{-T} \text { and } \quad \mathcal{O}=\frac{\mathrm{d} \omega}{\mathrm{d} \mathbf{F}} \mathbf{F}^{T}=-\xi \mathbf{I}
$$

for (90) and (50), respectively, while $\mathcal{A}^{d}$ vanishes for (49), the micro-stress $\mathbb{S}^{d}$ is a vector field, denoted by $\mathbf{z}^{d}$, and $\zeta^{d}, \bar{\gamma}^{d}$ are scalar fields.

Now, we are able to rewrite the condition (54) for a suspension of rigid granules by using relations (93)-(95),

$$
\begin{aligned}
& \operatorname{sym}\left[\mathbf{P}_{r} \mathbf{F}^{T}-\left(\xi \zeta_{r}^{d}+\nabla \xi \cdot \mathbf{z}_{r}^{d}\right) \mathbf{I}\right] \cdot \mathbf{D}-\xi\left(\mathbf{I} \oslash \mathbf{z}_{r}^{d}\right) \cdot \nabla \mathbf{D} \\
& +\mathbf{K}_{r}^{f} \cdot\left(\dot{\mathbf{R}}^{\prime}-\mathbf{Y} \mathbf{R}^{\prime}\right)+\mathbb{S}_{r}^{f} \cdot\left\{\nabla \dot{\mathbf{R}}^{\prime}+\left[\left(\nabla \mathbf{R}^{\prime}\right)^{T} \oslash \mathbf{Y}\right]^{T}\right\}=0
\end{aligned}
$$

or, better,

$$
\begin{aligned}
& \operatorname{sym}\left[\mathbf{P}_{r} \mathbf{F}^{T}-\left(\xi \zeta_{r}^{d}+\nabla \xi \cdot \mathbf{z}_{r}^{d}\right) \mathbf{I}\right] \cdot \mathbf{D}-\xi \mathbf{z}_{r}^{d} \cdot \nabla(\operatorname{tr} \mathbf{D}) \\
& +\left(\mathbf{K}_{r}^{f} \mathbf{R}^{\prime T}+\mathbb{S}_{r}^{f} \odot \nabla \mathbf{R}^{\prime}\right) \cdot\left(\dot{\mathbf{R}}^{\prime} \mathbf{R}^{\prime T}-\mathbf{Y}\right) \\
& +\left[\left(\mathbb{S}_{r}^{f}\right)^{t} \oslash \mathbf{R}^{\prime T}\right]^{t} \cdot \nabla\left(\dot{\mathbf{R}}^{\prime} \mathbf{R}^{\prime T}\right)=0
\end{aligned}
$$

where the usual properties of scalar products and derivations are used. This equation is identically verified for all totally free tensors $\mathbf{D} \in \operatorname{Sym}$ and $\left(\dot{\mathbf{R}}^{\prime} \mathbf{R}^{\prime T}-\mathbf{Y}\right) \in \mathrm{Skw}$, vector $\nabla(\operatorname{tr} \mathbf{D}) \in T_{x} \mathcal{B}$ and third-order tensor $\nabla\left(\dot{\mathbf{R}}^{\prime} \mathbf{R}^{\prime T}\right)$ skew-symmetric in the first two indices.

Therefore, it is 


$$
\begin{aligned}
& \mathbf{z}_{r}^{d}=\mathbf{0}, \quad \operatorname{sym}\left(\mathbf{P}_{r} \mathbf{F}^{T}-\xi \zeta_{r}^{d} \mathbf{I}\right)=\mathbf{O}, \\
& \operatorname{skw}\left(\mathbf{K}_{r}^{f} \mathbf{R}^{\prime T}+\mathbb{S}_{r}^{f} \odot \nabla \mathbf{R}^{\prime}\right)=\mathbf{O}, \quad 1 \operatorname{skw}\left[\left(\mathbb{S}_{r}^{f}\right)^{t} \oslash \mathbf{R}^{\prime T}\right]^{t}=\mathbf{O},
\end{aligned}
$$

where $\operatorname{lskw}(\cdot)$ indicates a third-rank tensor field skew in the first two indices, i.e., $(1 \mathrm{skw} \mathbb{\mathbb { T }})_{i j k}:=\frac{1}{2}\left(\mathbb{T}_{i j k}-\mathbb{\mathbb { T }}_{j i k}\right)$, and so

$$
\begin{aligned}
& \mathbf{z}_{a}^{d} \in T_{x} \mathcal{B}, \quad\left[\mathbf{P}_{a} \mathbf{F}^{T}-\left(\xi \zeta_{a}^{d}+\nabla \xi \cdot \mathbf{z}_{a}^{d}\right) \mathbf{I}\right] \in \mathrm{Sym}, \\
& \left(\mathbf{K}_{a}^{f} \mathbf{R}^{\prime T}+\mathbb{S}_{a}^{f} \odot \nabla \mathbf{R}^{\prime}\right) \in \mathrm{Skw}, \quad \mathbf{R}^{\prime}\left(\mathbb{S}_{a}^{f} \mathbf{w}\right)^{T} \in \mathrm{Skw}, \forall \text { vector } \mathbf{w} .
\end{aligned}
$$

Also now we split up the Capriz's balance (25) into a scalar equation, which governs the fluctuations of the volume fraction, and a tensor equation, which rules the micro-rotation rate of change, respectively:

$$
\bar{\gamma}^{d}-\zeta^{d}+\operatorname{Div} \mathbf{z}^{d}=0 \text { and } \overline{\mathbf{C}}^{f}-\mathbf{K}^{f}+\operatorname{Div} \mathbb{S}^{f}=\mathbf{O} .
$$

Using the principle (40) and the result (98) 1 , we have

$$
\zeta_{r}^{d}=\bar{\gamma}^{d}-\zeta_{a}^{d}+\operatorname{Div} \mathbf{z}_{a}^{d},
$$

while, proceeding as in Sect. 6.3 with the micro-rotation $\mathbf{R}^{\prime}$ instead of $\mathbf{G}$, we obtain

$$
\begin{aligned}
& \operatorname{skw}\left[\left(\overline{\mathbf{C}}^{f}-\mathbf{K}_{a}^{f}+\operatorname{Div} \mathbb{S}_{a}^{f}\right) \mathbf{R}^{\prime T}\right]=\mathbf{O} \text { and } \\
& \operatorname{sym}\left[\left(\overline{\mathbf{C}}^{f}-\mathbf{K}_{r}^{f}+\operatorname{Div} \mathbb{S}_{r}^{f}\right) \mathbf{R}^{\prime T}\right]=\mathbf{O},
\end{aligned}
$$

where results $(98)_{3,4}$ were used.

In the end, following the same procedure used for the relation (82) and also using the result $(98)_{2}$, we get the Piola-Kirchhoff stress tensor

$$
\begin{aligned}
\mathbf{P}= & {\left[\operatorname{sym}\left(\mathbf{P}_{a} \mathbf{F}^{T}\right)+\xi\left(\bar{\gamma}^{d}-\zeta_{a}^{d}+\operatorname{Div} \mathbf{z}_{a}^{d}\right) \mathbf{I}\right.} \\
& \left.+\operatorname{skw}\left(\mathbf{K}_{a}^{f} \mathbf{R}^{\prime T}+\mathbb{S}_{a}^{f} \odot \nabla \mathbf{R}^{\prime}\right)\right] \mathbf{F}^{-T}
\end{aligned}
$$

we then acquire the two pure balance equations of the rotational micro-momentum $(102)_{1}$ and macromomentum (19) in order to study the micro- and macro-motions of the suspension of rigid granules in a fluid matrix described in this subsection; here, the influence of its peculiar microstructural variables is put in evidence in the spherical and skew-symmetric parts of $\mathbf{P F}$ in the expression (103).

Furthermore, in addition to the relations (98), we also need Eqs. (101) and (102) 2 to obtain the expressions for reactions to the constraint.

Remark 6 The dilatant granular material with rotating rigid grains, presented in this example, could also be seen as a continuum with a spherical structure and, therefore, as a particular example of Sect. 6.3 (see, also, $[16,36])$. Precisely, introducing the following fields: the second-rank skew tensors of total external and internal rotational moments per unit volume $\overline{\mathbf{M}}$ and $\mathbf{N}$, respectively, and the third-order spinning hyperstress tensor $\mathbb{T}$, skew in the first two indices, defined as

$$
\begin{aligned}
\overline{\mathbf{M}} & :=\operatorname{skw}\left(\overline{\mathbf{C}}^{f} \mathbf{R}^{\prime}\right), \quad \mathbf{N}:=\operatorname{skw}\left(\mathbf{K}_{a}^{f} \mathbf{R}^{\prime T}+\mathbb{S}_{a}^{f} \odot \nabla \mathbf{R}^{\prime}\right), \\
\mathbb{T} \mathbf{w} & :=\operatorname{skw}\left[\left(\mathbb{S}_{a}^{f} \mathbf{w}\right) \mathbf{R}^{\prime T}\right], \forall \text { vector } \mathbf{w},
\end{aligned}
$$

we can rewrite the rotational micro-momentum balance $(102)_{1}$ in a more classical form

$$
\overline{\mathbf{M}}-\mathbf{N}+\operatorname{Div} \mathbb{T}=\mathbf{O},
$$

as it appears for the microrigid Cosserat continua (see equation (23.1) of [6] or equation (63) of [48]). In particular, if we decompose the volume external rotational moment $\overline{\mathbf{M}}$, as made in $\S 9$ of [36], in its inertial and non-inertial contributions as

$$
\overline{\mathbf{M}}=-\rho_{*} \mu_{*}^{2} \dot{\tilde{\mathbf{Y}}}+\rho_{*} \mathbf{M},
$$


where $\tilde{\mathbf{Y}}$ is the micro-spin tensor field defined in $(75)_{2}, \mu_{*}$ is a constant coefficient depending on the shape of the granules and $\mathbf{M}$ is the bulk non-inertial part, we have the most expressive formula

$$
\rho_{*} \mu_{*}^{2} \dot{\tilde{\mathbf{Y}}}=\rho_{*} \mathbf{M}-\mathbf{N}+\operatorname{Div} \mathbb{\pi} .
$$

\subsection{Pseudo-Cosserat continua}

In the classical case, studied by the Cosserat brothers [22], the affine microstructure is rigid in the sense that $v:=\mathbf{R}^{\prime}$, with $\mathbf{R}^{\prime}$ a proper orthogonal tensor. Here, we remove the rigidity constraint of the microdeformation, but we impose that the micro- and macro-motions have the same stretch, so we can use the procedure of Sect. 6.3.

In particular, if we use the right polar decomposition of the second-rank tensor field $\mathbf{G} \in \mathrm{Lin}^{+}$, which describes the local affine structure, i.e., $\mathbf{G} \equiv \mathbf{R}^{\prime} U^{\prime}$ with $\mathbf{R}^{\prime} \in \mathrm{Orth}^{+}$the micro-rotation and $\mathbf{U}^{\prime} \in \mathrm{Sym}^{+}$the right micro-stretch tensor such that $\mathbf{U}^{\prime}=\sqrt{\mathbf{G}^{T} \mathbf{G}}$, the partial holonomic constraint we consider here is such that the right micro- and macro-stretches $\mathbf{U}^{\prime}$ and $\mathbf{U}$, respectively, coincide.

Therefore, using the notations of Eq. (42), we pose

$$
v=\left(\frac{v^{f}}{v^{c}}\right):=\left(\frac{\mathbf{R}^{\prime}}{\mathbf{U}^{\prime}}\right)=\left(\frac{\mathbf{R}^{\prime}}{\mathbf{U}}\right) ;
$$

and, hence, for definition (8) the third-order tensor $\mathcal{A}^{f}$ and the constrained one $\mathcal{A}^{c}$ are now the following fields, in components,

$$
\mathcal{A}_{i J k}^{f}=\epsilon_{i l k} \mathrm{R}_{l J}^{\prime} \text { and } \mathcal{A}_{i J k}^{c}=0,
$$

because, for each two observer differing by a rotation $\mathbf{Q}$ in (7), different values of the order parameters are read: the proper orthogonal tensor $\mathbf{R}^{\prime}$ changes as follows $\mathbf{R}_{\mathbf{q}}^{\prime}=\mathbf{Q}^{T} \mathbf{R}^{\prime}$, while the right stretch is invariant, i.e., $\mathbf{U}_{\mathbf{q}}=\mathbf{U}$; we observe that $\mathcal{A}^{f}$ is like in (92).

Consequently, due to (110), the corotational derivatives are now

$$
\stackrel{\circ}{\mathbf{R}}^{\prime}=\dot{\mathbf{R}}^{\prime}-\mathbf{Y} \mathbf{R}^{\prime} \text { and } \quad \stackrel{\mathbf{U}}{=} \dot{\mathbf{U}},
$$

while the balance of moment of momentum (27) is obtained exactly as for (73) by replacing $\mathbf{G}$ with $\mathbf{R}^{\prime}$

$$
\operatorname{skw}\left(\mathbf{P} \mathbf{F}^{T}\right)=\operatorname{skw}\left[\mathbf{R}^{\prime}\left(\mathbf{K}^{f}\right)^{T}+\nabla \mathbf{R}^{\prime} \odot \mathbb{S}^{f}\right] .
$$

Finally, in the pseudo-Cosserat continua, proceeding as in (97), the expression (45) for the stress power $w$ reduces to

$$
\begin{aligned}
w= & \operatorname{sym}\left(\mathbf{P} \mathbf{F}^{T}\right) \cdot \mathbf{D}+\mathbf{K}^{c} \cdot \dot{\mathbf{U}}+\mathbb{S}^{c} \cdot \nabla \dot{\mathbf{U}}+\mathbf{K}^{f} \cdot\left(\dot{\mathbf{R}}^{\prime}-\mathbf{Y} \mathbf{R}^{\prime}\right) \\
& +\mathbb{S}^{f} \cdot\left\{\nabla \dot{\mathbf{R}}^{\prime}+\left[\left(\nabla \mathbf{R}^{\prime}\right)^{T} \oslash \mathbf{Y}\right]^{T}\right\}=\operatorname{sym}\left(\mathbf{P F}^{T}\right) \cdot \mathbf{D}+\mathbf{K}^{c} \mathbf{U} \cdot \dot{\mathbf{U}} \mathbf{U}^{-1} \\
& +\mathbb{S}^{c} \cdot \nabla \dot{\mathbf{U}}+\left(\mathbf{K}^{f} \mathbf{R}^{\prime T}+\mathbb{S}^{f} \odot \nabla \mathbf{R}^{\prime}\right) \cdot\left(\dot{\mathbf{R}}^{\prime} \mathbf{R}^{T}-\mathbf{Y}\right) \\
& +\operatorname{lskw}\left[\left(\mathbb{S}^{f}\right)^{t} \oslash \mathbf{R}^{\prime T}\right]^{t} \cdot \nabla\left(\dot{\mathbf{R}}^{\prime} \mathbf{R}^{\prime T}\right) \\
= & \operatorname{sym}\left[\left(\mathbf{P}+\mathbf{R} \mathbf{K}^{c}\right) \mathbf{F}^{T}\right] \cdot \mathbf{D}+\operatorname{skw}\left(\mathbf{R K}^{c} \mathbf{F}^{T}\right) \cdot \operatorname{skw}\left(\mathbf{R} \dot{\mathbf{U}} \mathbf{U}^{-1} \mathbf{R}^{T}\right) \\
& +\operatorname{lsym} \mathbb{S}^{c} \cdot \nabla \dot{\mathbf{U}}+\operatorname{skw}\left(\mathbf{K}^{f} \mathbf{R}^{\prime T}+\mathbb{S}^{f} \odot \nabla \mathbf{R}^{\prime}\right) \cdot\left(\dot{\mathbf{R}}^{\prime} \mathbf{R}^{\prime T}-\mathbf{Y}\right) \\
& +\operatorname{lskw}\left[\left(\mathbb{S}^{f}\right)^{t} \oslash \mathbf{R}^{\prime T}\right]^{t} \cdot \nabla\left(\dot{\mathbf{R}}^{\prime} \mathbf{R}^{\prime T}\right),
\end{aligned}
$$

where $\operatorname{lsym}(\cdot)$ indicates the left symmetry in the first two indices (as for lskw in (98)), while the following expression for the macro-stretching tensor $\mathbf{D}$ is used in the last row: $\mathbf{D}=\operatorname{sym}\left(\dot{\mathbf{F}} \mathbf{F}^{-1}\right)=\mathbf{R} \operatorname{sym}\left(\dot{\mathbf{U}}^{-1}\right) \mathbf{R}^{T}$, with $\mathbf{R}\left(=\mathbf{F} \mathbf{U}^{-1}\right)$ the rotation of the polar decomposition of the deformation gradient $\mathbf{F}$. 

tively:

As usual, we obtain the following results for reactions and actions of fields $\mathbf{P} \mathbf{K}^{c}, \mathbf{K}^{f}, \mathbb{S}^{c}$ and $\mathbb{S}^{f}$, respec-

$$
\begin{aligned}
& \operatorname{sym}\left[\left(\mathbf{P}_{r}+\mathbf{R} \mathbf{K}_{r}^{c}\right) \mathbf{F}^{T}\right]=\mathbf{O}, \quad \operatorname{skw}\left(\mathbf{R K}_{r}^{c} \mathbf{F}^{T}\right)=\mathbf{O}, \quad \operatorname{lsym} \mathbb{S}_{r}^{c}=\mathbf{O} \\
& \operatorname{skw}\left(\mathbf{K}_{r}^{f} \mathbf{R}^{\prime T}+\mathbb{S}_{r}^{f} \odot \nabla \mathbf{R}^{\prime}\right)=\mathbf{O}, \quad 1 \mathrm{skw}\left[\left(\mathbb{S}_{r}^{f}\right)^{t} \oslash \mathbf{R}^{\prime T}\right]^{t}=\mathbf{O}
\end{aligned}
$$

and

$$
\begin{aligned}
& \text { skw }\left[\left(\mathbf{P}_{a}+\mathbf{R} \mathbf{K}_{a}^{c}\right) \mathbf{F}^{T}\right]=\mathbf{O}, \quad \operatorname{sym}\left(\mathbf{R K}_{a}^{c} \mathbf{F}^{T}\right)=\mathbf{O}, \\
& \text { skw }\left(\mathbb{S}_{a}^{c} \mathbf{w}\right)=\mathbf{O}, \forall \text { vector } \mathbf{w}, \quad \operatorname{sym}\left(\mathbf{K}_{a}^{f} \mathbf{R}^{\prime T}+\mathbb{S}_{a}^{f} \odot \nabla \mathbf{R}^{\prime}\right)=\mathbf{O}, \\
& \operatorname{sym}\left[\left(\mathbb{S}_{a}^{f} \mathbf{w}\right) \mathbf{R}^{\prime T}\right]=\mathbf{O}, \forall \text { vector } \mathbf{w} .
\end{aligned}
$$

Again we use the splitting of the Capriz's balance (25) in order to obtain the appropriate set of pure equation; then we have

$$
\mathbf{K}_{r}^{f}-\operatorname{Div} \mathbb{S}_{r}^{f}=\overline{\mathbf{C}}^{f}-\mathbf{K}_{a}^{f}+\operatorname{Div} \mathbb{S}_{a}^{f} \text { and } \mathbf{K}_{r}^{c}=\overline{\mathbf{C}}^{c}-\mathbf{K}_{a}^{c}+\operatorname{Div} \mathbb{S}_{a}^{c},
$$

where all the tensors in the second equation, which regulates the rate of change of the micro-elongation $\mathbf{U}$, are symmetric and we applied (114) $)_{3}$; so, multiplying the two sides of Eq. (116) from the right by the operator $\mathbf{R}^{\prime T}$, considering the symmetric and skew parts of them and using relations $(114)_{4,5}$ and $(115)_{4,5}$, respectively, we have

$$
\begin{aligned}
& \operatorname{skw}\left[\left(\overline{\mathbf{C}}^{f}-\mathbf{K}_{a}^{f}+\operatorname{Div} \mathbb{S}_{a}^{f}\right) \mathbf{R}^{\prime T}\right]=\mathbf{O} \text { and } \\
& \operatorname{sym}\left[\left(\mathbf{K}_{r}^{f}-\operatorname{Div} \mathbb{S}_{r}^{f}\right) \mathbf{R}^{\prime T}\right]=\operatorname{sym}\left(\overline{\mathbf{C}}^{f} \mathbf{R}^{\prime T}\right) ;
\end{aligned}
$$

similarly, making the same with equation $(116)_{2}$ by multiplying the two side from the left by the operator $\mathbf{R}$ and from the right by $\mathbf{F}^{T}$, we obtain

$$
\operatorname{sym}\left(\mathbf{R K}_{r}^{c} \mathbf{F}^{T}\right)=\operatorname{sym}\left[\mathbf{R}\left(\overline{\mathbf{C}}^{c}+\operatorname{Div} \mathbb{S}_{a}^{c}\right) \mathbf{F}^{T}\right]
$$

for relations $(114)_{3}$ and $(115)_{2}$.

The macro-motion is always governed by the Cauchy equation (19), with the Piola-Kirchhoff stress tensor given by

$$
\begin{aligned}
\mathbf{P F}^{T}= & \operatorname{sym}\left(\mathbf{P F}^{T}\right)+\operatorname{skw}\left(\mathbf{P} \mathbf{F}^{T}\right)=\operatorname{sym}\left(\mathbf{P}_{a} \mathbf{F}^{T}\right)+\operatorname{sym}\left(\mathbf{P}_{r} \mathbf{F}^{T}\right) \\
& +\operatorname{skw}\left[\mathbf{R}^{\prime}\left(\mathbf{K}^{f}\right)^{T}+\nabla \mathbf{R}^{\prime} \odot \mathbb{S}^{f}\right] \\
= & \operatorname{sym}\left(\mathbf{P}_{a} \mathbf{F}^{T}\right)-\operatorname{sym}\left(\mathbf{R} \mathbf{K}_{r}^{c} \mathbf{F}^{T}\right)-\operatorname{skw}\left[\mathbf{K}_{a}^{f} \mathbf{R}^{\prime T}+\mathbb{S}_{a}^{f} \odot \nabla \mathbf{R}^{\prime}\right] \\
= & \operatorname{sym}\left\{\left[\mathbf{P}_{a}-\mathbf{R}\left(\overline{\mathbf{C}}^{c}+\operatorname{Div} \mathbb{S}_{a}^{c}\right)\right] \mathbf{F}^{T}\right\}-\operatorname{skw}\left[\overline{\mathbf{C}}^{f} \mathbf{R}^{\prime T}+\nabla\left(\mathbb{S}_{a}^{f} \oslash \mathbf{R}^{\prime T}\right)\right],
\end{aligned}
$$

where the moment of momentum balance (112) was used in the first row, relations (114) $)_{1,4}$ in the second and balances (117) ${ }_{1}$ and (118) in the last. This pure Cauchy equation, together with the pure balance for the rotational micro-momentum (117) 1 , rules the mechanical behavior of the pseudo-Cosserat medium on the macro- and micro-scale, respectively. As usual the reactions could be obtained from the remaining dynamical equations (116) 2 and (117) 2 .

Remark 7 An expression like Eq. (108) could be easily obtained also here, instead we want to focus on the expression of the Piola-Kirchhoff stress tensor $\mathbf{P}$ in $(119)_{5}$ and observe once again the lack of symmetry of the Cauchy tensor $\mathbf{T}$ and its possible dependence on higher-order derivatives without incurring certain apparent inconsistencies with rational thermodynamics, when constitutive laws are considered (see, e.g., [5] or [41]).

Remark 8 The pseudo-Cosserat continuum is a generalization of the classical model of Cosserat brothers [22] that we could easily recover from our model when the right micro-stretch tensor coincides with the identity tensor, i.e., $\mathbf{U}^{\prime}=\mathbf{I}$. 


\section{Closing remarks}

The proposals and developments of the preceding paragraphs may appear to be nothing more than a speculative exercise with no practical value. So perhaps it is deserving to show that some other continuum models currently in use are special cases of partially constrained continua and that they presuppose, albeit tacitly, the properties made explicit here.

For example, the generally accepted model for an incompressible continuum with finely dispersed cavities, e.g., a liquid containing compressible gas bubbles [8], or an elastic solid with nano-pores filled with an inviscid fluid [35], can be inserted in the class of partially constrained continua, as well as the classical Grioli-Toupin theory $[47,66]$ which can therefore be derived from an affine microstructured body by requiring the microspin to be equal to the macro-one, or yet the smectic-C liquid crystal elastomers which are layered materials exhibiting a solid-like elastic response along the normal to the layer, and a rubbery one in the plane, hence possess microstructures both of the material and local type (the nematic microstructure and the lamellae, respectively) represented with a vectorial microstructure partially constrained (see $[3,38])$.

Many other topics deserve to be analyzed and have not been touched upon in this work. The main ones, which we intend to consider in a future paper, are the study of the contributions of inertia to external macro- and microforces of mass in the pure equations of motion and, moreover, the generalization to internal thermodynamical constraints, in order to develop a complete constitutive theory.

Acknowledgements This research is part of the activities of the PRIN Project 2017 J4EAYB: "Multiscale innovative materials and structures (MIMS)." The support of the "Gruppo Nazionale di Fisica Matematica" of the "Istituto Nazionale di Alta Matematica 'F. Severi' (GNFM-INDAM)" is also recognized.

Funding Open access funding provided by Universitá degli Studi Mediterranea di Reggio Calabria within the CRUI-CARE Agreement.

Open Access This article is licensed under a Creative Commons Attribution 4.0 International License, which permits use, sharing, adaptation, distribution and reproduction in any medium or format, as long as you give appropriate credit to the original author(s) and the source, provide a link to the Creative Commons licence, and indicate if changes were made. The images or other third party material in this article are included in the article's Creative Commons licence, unless indicated otherwise in a credit line to the material. If material is not included in the article's Creative Commons licence and your intended use is not permitted by statutory regulation or exceeds the permitted use, you will need to obtain permission directly from the copyright holder. To view a copy of this licence, visit http://creativecommons.org/licenses/by/4.0/.

\section{Declarations}

Conflict of interest The author declares that he has no conflict of interest.

\section{References}

1. Ahmadi, G.: A generalized continuum theory for granular materials. Int. J. Non-Linear Mech. 17, 21-33 (1982)

2. Amoddeo, A., Giovine, P.: Micromechanical modelling of granular materials and FEM simulations. Meccanica 54, 609-630 (2019)

3. Buonsanti, M., Giovine, P.: On a Minimum problem in smectic elastomers. In: Santini, A., Moraci, N. (eds.) 2008 Seismic Engineering Conference: Commemorating the 1908 Messina and Reggio Calabria Earthquake. American Institute of Physics Conference Proceedings, vol. 1020, pp. 1350-1357. AIP, New York (2008)

4. Capriz, G.: Continua with latent microstructure. Arch. Ration. Mech. Anal. 90, 43-56 (1985)

5. Capriz, G.: Continua with constrained or latent microstructure. In: Ball, J.M. (ed.) Material Instabilities in Continuum Mechanics and Related Mathematical Problems, pp. 53-64. Oxford Univ. Press, Oxford (1988)

6. Capriz, G.: Continua with Microstructure. Springer Tracts in Natural Philosophy, vol. 35. Springer, New York (1989)

7. Capriz, G.: Continua with Substructure. Phys. Mesomech. 3(5-14), 37-48 (2000)

8. Capriz, G., Cohen, H.: The bubbly fluid as a continuum with microstructure. Mech. Res. Commun. 10, 359-367 (1983)

9. Capriz, G., Giovine, P.: On microstructural inertia. Math. Mod. Methods Appl. Sci. 7, 211-216 (1997)

10. Capriz, G., Giovine, P.: Remedy to omissions in a tract on continua with microstructure. Atti del XIII Congresso Nazionale di Meccanica Teorica e Applicata, General Mechanics, AIMETA'97, Siena, vol. I, pp. 1-6 (1997)

11. Capriz, G., Giovine, P.: Weakly nonlocal effects in mechanics. In: Contributions to Continuum Theories, Krzysztof Wilmanski's Anniversary Volume, Weierstrass Institute for Applied Analysis and Stochastics, Berlin, Report n. 18 - ISSN 0946-8838, pp. 37-44 (2000)

12. Capriz, G., Giovine, P.: Classes of ephemeral continua. Math. Methods Appl. Sci. 41, 1175-1196 (2018)

13. Capriz, G., Mazzini, G.: Invariance and balance in structured continua. ZAMM 76(S4), 121-124 (1996)

14. Capriz, G., Mazzini, G.: Invariance and balance in continuum mechanics. In: Butazzo, G., Galdi, G.P., Lanconelli, E., Pucci, P. (eds.) Nonlinear Analysis and Continuum Mechanics, pp. 27-35. Springer, New York (1998) 
15. Capriz, G., Podio Guidugli, P.: Formal structure and classification of theories of oriented materials. Ann. Mat. Pura Appl. (IV) CXV, 17-39 (1977)

16. Capriz, G., Podio Guidugli, P.: Materials with spherical structure. Arch. Ration. Mech. Anal. 75, 269-279 (1981)

17. Capriz, G., Podio Guidugli, P.: Internal constraints. In: Truesdell, C. (ed.) Rational Thermodynamics, 2nd edn., pp. 159-170. Springer, New York (1984)

18. Capriz, G., Trebeschi, P.: Reflections upon the axioms of continuum mechanics prompted by the study of complex materials. Bull. Tech. Univ. Istanb. 47, 1-12 (1994)

19. Capriz, G., Virga, E.G.: Interactions in continua with microstructure. Arch. Ration. Mech. Anal. 109, 323-342 (1990)

20. Capriz, G., Virga, E.G.: On singular surfaces in the dynamics of continua with microstructure. Quart. Appl. Math. 52, 509-517 (1994)

21. Chen, K.C., Lan, J.Y., Tai, Y.C.: Description of local dilatancy and local rotation of granular assemblies by microstretch modeling. Int. J. Sol. Struct. 46, 3882-3893 (2009)

22. Cosserat, E.F.: Théorie des Corps Déformables. Hermann, Paris (1909)

23. Degiovanni, M., Marzocchi, A., Musesti, A.: Cauchy fluxes associated with tensor having divergence measure. Arch. Ration. Mech. Anal. 147, 197-223 (1999)

24. Demiray, H.: A continuum theory of diatomic solids: viewed as directed media. J. Eng. Math. 11, 257-271 (1977)

25. Di Carlo, A.: A non-standard format for continuum mechanics. In: Batra, R.C., Beatty, M.F. (eds.) Contemporary Research in the Mechanics and Mathematics of Materials, pp. 92-104. CIMNE, Barcelona (1996)

26. Dinculeanu, N.: Vector Measures. Pergamon Press, Berlin (1967)

27. Dunn, J.E.: Interstitial working and a nonclassical continuum thermodynamics. In: Serrin, J. (ed.) New Perspectives in Thermodynamics, pp. 187-222. Springer, Berlin-Heidelberg (1986)

28. Dunn, J.E., Serrin, J.: On the thermomechanics of interstitial working. Arch. Ration. Mech. Anal. 88, 95-133 (1985)

29. Eringen, A.C.: Mechanics of micromorphic continua. In: Kröner, E. (ed.) Proceedings of the IUTAM Symposium on Mechanics of Generalized Continua. Freudenstadt and Stuttgart 67, pp. 18-35. Springer, Berlin-Heidelberg-New York (1968)

30. Focardi, M., Mariano, P.M., Spadaro, E.N.: Multi-value microstructural descriptors for complex materials: analysis of ground states. Arch. Ration. Mech. Anal. 217(3), 899-933 (2015)

31. Giovine, P.: Porous solids as materials with ellipsoidal structure. In: Batra, R.C., Beatty, M.F. (eds.) Contemporary Research in the Mechanics and Mathematics of Materials, pp. 335-342. CIMNE, Barcelona (1996)

32. Giovine, P.: Nonclassical thermomechanics of granular materials. Math. Phys. Anal. Geom. 2, 179-196 (1999)

33. Giovine, P.: A continuum description of diatomic systems. In: Brocato, M., Podio Guidugli, P. (eds.) Rational Continua, Classical and New, pp. 97-109. Springer, Milano (2003)

34. Giovine, P.: A continuum theory of soils: viewed as peculiar immiscible mixtures. Math. Comput. Model. 37, 525-532 (2003)

35. Giovine, P.: On adsorption and diffusion in microstructured porous media. In: Huyghe, J.M., Raats, P.A.C., Cowin, S.C. (eds.) IUTAM Symposium on Physico-Chemical and Electromechanical Interactions in Porous Media. Series on Solid Mechanics and Its Applications, vol. 125, pp. 183-191. Springer, Dordrecht (2005)

36. Giovine, P.: An extended continuum theory for granular media. In: Capriz, G., Giovine, P., Mariano, P.M. (eds.) Mathematical Models of Granular Matter. Series: Lecture Notes in Mathematics, vol. 1937, pp. 167-192. Springer, Berlin (2008)

37. Giovine, P.: Remarks on constitutive laws for dry granular materials. In: Giovine, P., Goddard, J.D., Jenkins, J.T. (eds.) IUTAM-ISIMM Symposium on Mathematical Modeling and Physical Instances of Granular Flows. AIP Conference Proceedings Series, pp. 314-322. AIP, New York (2010)

38. Giovine, P.: On constitutive choices for smectic elastomers. In: Albers, B. (ed.) Continuous Media with Microstructure, Collection in honor of Krzysztof Wilmanski on the Occasion of his 70th Birthday, pp. 69-79. Springer, Berlin (2010)

39. Giovine, P.: Extended Granular Micromechanics. In: Radjai, F., Nezamabadi, S., Luding, S., Delenne, J.Y. (eds.) Powders and Grains 2017-8th International Conference on Micromechanics on Granular Media, France: EPJ Web of Conferences, vol. 140, pp. 11009 (2017). https://doi.org/10.1051/epjconf/201714011009

40. Giovine, P.: A multiscale approximation method to describe diatomic crystalline systems: constitutive equations. J. Multiscale Model. 9(3), 1840001 (2018). ((13 pages))

41. Giovine, P.: Notes on constitutive relations for porous solids. In: Giovine, P., Mariano, P.M., Mortara, G. (eds.) Views on Microstructures in Granular Materials. Series: Advances in Continuum Mechanics, vol. 44, pp. 61-85. Birkhauser, Basel (2020)

42. Giovine, P.: Internal constraints in the theories of immiscible mixtures for soils. Int. J. Solids Struct. 187, 3-22 (2020)

43. Giovine, P., Margheriti, L., Speciale, M.P.: On wave propagation in porous media with strain gradient effects. Comp. Math. Appl. 55, 307-318 (2008)

44. Giovine, P., Oliveri, F.: Dynamics and wave propagation in dilatant granular materials. Meccanica 30, 341-357 (1995)

45. Goodman, M.A., Cowin, S.C.: A continuum theory for granular materials. Arch. Ration. Mech. Anal. 44, 249-266 (1972)

46. Green, A.E., Naghdi, P.M., Trapp, J.A.: Thermodynamics of a continuum with internal constraints. Int. J. Eng Sci. 8, 891-908 (1970)

47. Grioli, G.: EIasticità asimmetrica. Ann. Matem. Pura Appl. 4(50), 387-417 (1960)

48. Grioli, G.: Microstructures as a refinement of Cauchy theory. Problems of physical concreteness. Cont. Mech. Thermodyn. 15, 441-450 (2003)

49. Gurtin, M.E., Podio Guidugli, P.: The thermodynamics of constrained materials. Arch. Ration. Mech. Anal. 51, 192-208 (1973)

50. Ichikawa, Y., Selvadurai, A.P.S.: Transport Phenomena in Porous Media: Aspects of Micro/Macro Behaviour. Springer, Berlin, Heidelberg (2012)

51. Kanatani, K.I.: A micropolar continuum theory for the flow of granular materials. Int. J. Eng. Sci. 17, 419-432 (1979)

52. Lee, J.M.: Introduction to Smooth Manifolds. Springer Graduate Texts in Mathematics, vol. 218. Springer, Berlin, New York (2003)

53. Liu, I.S.: Method of Lagrange multipliers for exploitation of the entropy principle. Arch. Ration. Mech. Anal. 46, 131-148 (1972) 
54. Mariano, P.M.: Multifield theories in mechanics of solids. Adv. Appl. Mech. 38, 1-93 (2002)

55. Mariano, P.M., Stazi, F.L.: Computational aspects of the mechanics of complex materials. Arch. Comput. Methods Eng. 12(4), 391-478 (2005)

56. Mariano, P.M.: Cracks in complex bodies: covariance of tip balances. J. Nonlinear Sci. 18, 99-141 (2008)

57. Mariano, P.M.: Mechanics of material mutations. Adv. Appl. Mech. 47, 1-91 (2014)

58. Noll, W.: La Mécanique Classique, Basée sur un Axiome d'Objectivité. La Méthode Axiomatique dans les Mécaniques Classiques et Nouvelles, (Colloque International, Paris, 1959), Gauthiers-Villars, Paris, 47-56 (1963). [Reprinted in The Foundations of Mechanics and Thermodynamics, Selected Works by W. Noll \& C. A. Truesdell (Eds.), Springer, Berlin, 135-144 (1974)]

59. Noll, W., Virga, E.G.: Fit regions and functions with bounded variation. Arch. Ration. Mech. Anal. 102, 1-21 (1988)

60. Noll, W., Virga, E.G.: On edge interactions and surface tensions. Arch. Ration. Mech. Anal. 111, 1-31 (1990)

61. Nunziato, J.W., Cowin, S.C.: A nonlinear theory of elastic materials with voids. Arch. Ration. Mech. Anal. 72, 175-201 (1979)

62. Reynolds, O.: On the dilatancy of media composed of rigid particles in contact. Philos. Mag. 20, 469-481 (1885)

63. Segev, R.: Forces and the existence of stresses in invariant continuum mechanics. J. Math. Phys. 27, 163-170 (1986)

64. Segev, R.: A geometrical framework for the static of materials with microstructure. Math. Mod. Methods Appl. Sci. 4, 871-897 (1994)

65. Segev, R.: Fluxes and flux-conjugated stresses. In: Capriz, G., Mariano, P.M. (eds.) Advances in Multifield Theories of Continua with Substructure, pp. 149-163. Birkhäuser, Boston (2004)

66. Toupin, R.A.: Elastic materials with couple-stresses. Arch. Ration. Mech. Anal. 11, 385-414 (1962)

67. Truesdell, C.: A First Course in Rational Continuum Mechanics, vol. 1. Academic Press, New York (1977)

68. Truesdell, C., Noll, W.: The non-linear field theories of mechanics. In: Flügge, S. (ed.) Handbuch der Physik, III/3. Springer, Berlin-Heidelberg-New York (1965)

69. Truesdell, C., Toupin, R.A.: The classical field theories. In: Flügge, S. (ed.) Handbuch der Physik, III/1. Springer, Berlin (1960)

Publisher's Note Springer Nature remains neutral with regard to jurisdictional claims in published maps and institutional affiliations. 